Fayetteville State University

DigitalCommons@Fayetteville State University

Math and Computer Science Faculty Working

Papers

Math and Computer Science

$10-2014$

\title{
Generalized Theoretical Criteria for Annihilation of HIV-1 Virions during HAART
}

Frank Nani

Fayetteville State University, fnani@uncfsu.edu

Mingxian Jin

Fayetteville State University, mjin@uncfsu.edu

Follow this and additional works at: https://digitalcommons.uncfsu.edu/macsc_wp

Part of the Computer Sciences Commons, Diseases Commons, Life Sciences Commons, and the Mathematics Commons

\section{Recommended Citation}

Nani, Frank and Jin, Mingxian (2015). "Generalized Theoretical Criteria for Annihilation of HIV-1 Virions during HAART", British Journal of Mathematics \& Computer Science (2): 262-299, 2015, Article no.BJMCS.2015.018. ISSN: 2231-0851. Print

This Article is brought to you for free and open access by the Math and Computer Science at DigitalCommons@Fayetteville State University. It has been accepted for inclusion in Math and Computer Science Faculty Working Papers by an authorized administrator of DigitalCommons@Fayetteville State University. For more information, please contact dballar5@uncfsu.edu. 
British Journal of Mathematics \& Computer Science

5(2): 262-299, 2015, Article no.BJMCS.2015.018

ISSN: 2231-0851

SCIENCEDOMAIN international

wWW.sciencedomain.org

\title{
Generalized Theoretical Criteria for Annihilation of HIV-1 Virions during HAART
}

\author{
Frank Nani ${ }^{*}$ and Mingxian Jin ${ }^{1}$ \\ ${ }^{I}$ Department of Mathematics and Computer Science, Fayetteville State University, 1200 \\ Murchison Road, Fayetteville, NC 28301, USA.
}

Article Information

DOI: $10.9734 / \mathrm{BJMCS} / 2015 / 11394$

Editor(s):

(1) Carlo Bianca, Department of Mathematical Sciences, Polytechnic University of Turin, Torino, Italy. (2) Tian-Xiao He, Department of Mathematics and Computer Science, Illinois Wesleyan University, USA.

Reviewers:

1. Anonymous, Morocco.

(2) Isack Kibona, Dept. of Science and Business Management, Mbeya University of Science and Technology, Tanzania.

(3) Rachel Waema Mbogo, Strathmore Univ., Center for Applied Research in Mathematical Sciences (CARMS), Kenya.

(4) Anonymous, Yuncheng University, P.R. China.

(5) Anonymous, Xi' an Jiaotong University, P.R.China.

Complete Peer review History: http://www.sciencedomain.org/review-history.php?iid=726\&id=6\&aid=6735

Original Research Article

Received: 13 May 2014

Accepted: 04 October 2014

Published: 30 October 2014

\section{Abstract}

Aims: This paper is an elaborate and quantitative attempt to construct medically applicable mathematical models and derive criteria for efficacious Highly Active Anti-Retroviral Therapy (HAART) protocol for an AIDS patient. The patho-physiological dynamics of Human Immuno-deficiency Virus type 1 (HIV-1) induced AIDS during HAART is modeled by a system of non-linear deterministic differential equations. The physiologically relevant and clinically plausible equations depict the dynamics of uninfected CD4 $4^{+} \mathrm{T}$ cells $\left(x_{1}\right), \mathrm{HIV}-1$ infected $\mathrm{CD}^{+}$T cells $\left(x_{2}\right)$, HIV-1 virions in the blood plasma $\left(x_{3}\right), \mathrm{HIV}-1$ specific $\mathrm{CD} 8^{+} \mathrm{T}$ cells $\left(x_{4}\right)$, and the concentration of HAART drug molecules $\left(x_{5}\right)$. The major objective of this research is to construct an elaborate mathematical model that depicts patho-physiodynamics of HIV-1 virions during HAART. The derived therapeutic criteria are expressed in terms of clinically measurable physiological parameters. Investigative computer simulations which describe certain aspects of HIV-1 dynamics during HAART are also presented in the paper.

Study Design: The mathematical model is constructed based on contemporary research data condensed from the clinical literature on HAART of AIDS. A system of coupled non-linear deterministic differential equations are used to characterize the patho-physiodynamics of

*Corresponding author: fnani@uncfsu.edu; 
HAART during the post chronic phase. The mathematical analysis of the model equations and the computer simulations are performed with regard to HAART protocols with constant continuous intravenous and transdermal drug infusions. A syncytium term with stoichiometric coefficient is introduced into the model to account for the formation of large multinucleated gp120 bearing $\mathrm{CD}^{+} \mathrm{T}$ cells that are observed in some AIDS patients. By assigning a zero value to the stoichiometric coefficient, the role of syncytium is abrogated.

Place and Duration of Study: This research was done at Fayetteville State University, North Carolina USA, and is sponsored by the FSU Mini-Grant Award and the HBCU Graduate STEM Grant. The research was conducted during the Spring of 2012.

Methodology: The deterministic nonlinear dynamics of HIV-1 AIDS equations are analyzed using the techniques of dynamical system theory, principles of linearized stability, non-linear system theory, and other relevant mathematical techniques. The clinically desirable equilibrium states, their local existence, and global stability are analyzed. Investigative computer simulations are performed illustrating some physiological outcomes.

Results: The therapeutic outcomes are presented in the form of theoretical criteria which are obtained from mathematical analysis of the model equations. In particular, the critical parameters which govern the dynamics of HIV-1 virions during HAART are clearly identified. Some clinical implications of HAART are elucidated in the computer simulations using hypothetical physiological parametric configurations.

Conclusion: This research has demonstrated the existence of plausible criteria under which HIV-1 virions can be annihilated using HAART. The latent HIV-1 virion reservoirs are implicated in unsuccessful scenarios of HAART. It has also been demonstrated that the rate constants associated with activation of lymphocytes by cytokine interleukin-2 (IL-2) play a significant role in determining the efficacious outcomes of HAART.

Keywords: HIV-1 patho-physiodynamics, mathematical modeling, HAART therapy, AIDS cure criteria, Michaelis-Menten kinetics.

AMS Subject Classification: 93A30; 93D05; 93D20; 34A34; 92C42; 92C35.

\section{Introduction}

Highly Active Anti-retroviral Therapy (HAART) is currently the most therapeutically efficacious treatment protocol for treating the Acquired Immunodeficiency Syndrome (AIDS). HIV-1 virions induce AIDS by orchestrating an irreversible destruction of the $\mathrm{CD} 4^{+} \mathrm{T}$ cells which then paralyze the immune system of the HIV-1 positive person. The major objectives of HAART therapy are the prolongation and improvement of the long-term life quality of patients; optimization of therapy such as to suppress the HIV-1 viral load to below 50 copies of HIV-1 RNA; reconstitution of the patients' immune system such that the $\mathrm{CD}^{+} \mathrm{T}$ cells proliferate to carrying capacity; and minimization of drug toxicity. HAART treatment protocol consists of nucleoside reverse transcriptase inhibitors, non-nucleoside reverse transcriptase inhibitors, protease inhibitors, antifungals /anti-bacterials and in future, integrase inhibitors. The reverse transcriptase inhibitors prevent reverse transcription of HIV-1 specific DNA. The protease inhibitors are antagonistic to maturation and formation of new HIV-1 virions. The possible role of integrase inhibitors is to prevent the integration of HIV-1 viral DNA into the patients' DNA [1,2].

In order to achieve the therapeutic goals of HAART, it is plausible to involve the techniques of mathematical modeling. Before the advent of HAART, the primary focus of the mathematical modelers is to quantitatively analyze the observed patho-physiodynamics of HIV-1 infection in 
the AIDS patients. These earlier research papers involve the pioneering work of Perelson et al. [3], Nowark et al. [4], and other contributors [5,6].

A recent paper by Nani and Jin [7] provided some physiological criteria under which HIV-1 virions in an AIDS patient can be annihilated during HAART.

Some of the earlier mathematical modeling publications focus on single-drug AIDS therapy using Zidovudine (INN) or Azidothymidine (AZT) [8]. Then the advent of Active Retro-viral Therapy (ART) and the associated clinical limitation let to the development of HAART treatment protocols. In spite of the initial success of HAART, there are clinically measurable and observable shortcomings in the treatment of AIDS [9]. In particular, HAART is not successful in about $40 \%$ of AIDS patients because of drug-induced toxicity and complications of treatment. HAART protocols have been clinically observed to have limited therapeutic efficacy due to biochemical/clinical drug resistance, short drug half-life, low bio-availability and blood plasma toxicities.

Mathematical modeling provides a quantitative and rational approach to solve the therapeutic efficacy problems associated with HAART. In particular, the models focused on finding optimal therapeutic schedules, the roles of latent viral reservoirs as well as minimizing of toxic side effect $[5,6,8,10-14]$.

Mathematical models which incorporate the explicit computation of the cure rate of HIV-1 induced AIDS during HAART include models by [15-17].

During HAART, the major obstacle to total viral eradication is the existence of viral reservoirs and retroviral latency [18]. Retroviral latency is defined as an integrated pro-virus with no active transcription. This is associated with low-level chronic viral and persistent cryptic HIV-1 replication. These processes lead to viral persistence after initial successful HAART [18]. Mathematical models which demonstrate persistence due to viral reservoirs have been constructed by Nani and Jin [15,16]. Other models which elegantly address the role of viral reservoirs in persistence include papers by Rong and Perelson in [19], Kim and Perelson in [20], and Sedaghat, Siliciano and Wilke in [21].

Optimal therapies that will minimize side effects have been investigated by many authors in [3-5,7,8,14,22,23]. Zaric et al. in 1998 [24] presented a model which was focused on the simulation of protease inhibitors and role of drug resistant HIV-1 virions. Stengel in [22] presented a mathematical model of HIV-1 infection and HAART which demonstrated the efficacy of a mathematically optimal therapy. Caetano and Yoneyama in [11] constructed a HAART model which incorporated the roles of latently infected $\mathrm{CD}^{+} \mathrm{T}$ cells, and discussed how the reverse transcriptase and protease inhibitors affected HIV-1 dynamics during HAART, using the LQR, Scheme. In particular, Hattaf and Yousfi incorporated the basic reproductive number $R_{0}$ in determination of optimal HIV therapy in [25]. In a separate paper, the same authors constructed a delayed differential equation model of HIV-1 dynamics during therapy in [26]. They employed a numerical algorithm and Pontryagin's Maximum Principle in determination of the optimal therapies. In a future paper, we will use Pontryagin's Minimal Principle to construct admissible optimal therapies such as to minimize the toxicity of the drug but maximize the therapeutic efficacy of HAART. 
In this paper, an elaborate mathematical model will be constructed which will incorporate physiologically plausible effects such as Michaelis-Menten kinetics, role of HIV-1 latent viral reservoirs, continuous transdermal drug delivery, and the implicit lymphocyte proliferation induction by the $\mathrm{CD}^{+} \mathrm{T}$ cells. The activation and proliferation is accomplished by a paracrine and autocrine processes which are mediated by the cytokine interleukin-2, secreted by the $\mathrm{CD} 4^{+} \mathrm{T}$ cells. A special feature of the current paper is the inclusion of the syncytium production term to the HIV-1 virion compartment. It has been shown by Koot et al. [27] in that syncytium inducing HIV-1 variants, occurring 2 years before progression to AIDS, on an average, significantly increase the rate of CD4+ declines. The destruction mechanism of CD4+ and CD8+ T cells due to syncytium formation and other mechanisms has been described by Shut et al. [28]. These features are incorporated in the current paper by assigning two separate cell loss rates. The HIV virions can also disintegrate by releasing the soluble HIV-1 envelope protein gp120 into the blood plasma which can attach to uninfected CD4+ T cells, causing attack by antibodies thereby increasing the rate of decline of immuno-competent $\mathrm{CD} 4+\mathrm{T}$ cells. Mathematical structures are used in the current model to demonstrate these aspects of HIV-1 and CD4+ T cell dynamics.

Several authors investigated the consequences of structured long-term and short-term treatment interruptions during HAART [9-11,14]. The current model will discuss these consequences by means of simulations.

The current paper will be divided into eight sections. The first section gives the introduction into HAART therapy and provides the basis for current research. This is followed by presentation and discussion of the model parameters in Section 2. In Section 3 the mathematical model of HAART therapy will be constructed. Also the necessary and sufficient criteria for annihilation of HIV-1 virions during HAART will be presented in sections 4, 5, and 6. In Section 7, clinically plausible computer simulations will be exhibited. Section 8 will conclude the paper.

\section{Parameters}

In this section, the physiological variable and parameters of the HAART model equations will be defined and explained. It must be emphasized that some of these parameters are biologically measurable or can be estimated using clinical techniques. In clinical experience, these parameters are different from patient to patient depending on their patho-physiological conditions.

A list of model parameters, constants, and variables is shown as follows.

$x_{1}$ : The number density of non-HIV-1-infected CD4 ${ }^{+}$helper T-lymphocytes per unit volume

$x_{2}$ : The number density of HIV-1 infected CD4 ${ }^{+}$helper T-lymphocytes per unit volume

$x_{3}$ : The number density of HIV-1 virions in the blood plasma per unit volume

$x_{4}$ : The number density of HIV-1 specific CD8 ${ }^{+}$cytotoxic T-lymphocytes per unit volume

$x_{5}$ : The concentration of drug molecules of the HAART treatment protocol

$S_{1} \quad$ : Rate of supply of un-infected $\mathrm{CD}^{+} \mathrm{T}_{4}$-lymphocytes

$S_{2} \quad$ : Rate of supply of latently infected $\mathrm{CD}^{+} \mathrm{T}_{4}$-lymphocytes

$S_{3}$ : Rate of supply of HIV-1 virions from macrophage, monocytes, microglial cells and other lymphoid tissue different from $\mathrm{T}_{4}$-lymphocytes

$S_{4} \quad$ : Rate of supply of $\mathrm{CD}^{+} \mathrm{T}_{8}$ lymphocytes from the thymus

$D$ : Rate of HAART drug infusion by transdermal delivery 
$a_{i}, b_{i}$ : Constant associated with activation of lymphocytes by cytokine interleukin-2 (IL-2) ( $i$ $=1,2,3,4)$

$c \quad$ : Rate of HAART drug degradation and excretion

$\alpha_{i}$ : Constant associated with HIV-1 infection of $\mathrm{CD}^{+} \mathrm{T}_{4}$ helper cells $(i=1,2,3)$

$\beta_{1}$ : The number of HIV-1 virions produced per day by replication and budding in $\mathrm{CD}^{+} \mathrm{T}_{4}$ helper cells

$\beta_{2}$ : Rate constant associated with replication and "budding" of HIV-1 in syncytia CD4 $4^{+} \mathrm{T}_{4}$ helper cells per day per microliter $(\mu l)$ and released into the blood plasma

$\beta_{3}$ : The number of HIV-1 virionsproduced per day by replication and "budding" in nonsyncytia $\mathrm{CD}^{+} \mathrm{T}_{4}$ helper cells and released into the blood plasma

$\eta_{i}$ : Constant depicting the rate of which HIV-1 virions incapacitate the $\mathrm{CD} 8^{+} \mathrm{T}_{8}$ cytotoxic cells $(i=1,2)$

$\left(\sigma_{0}, \lambda_{0}\right)$ : Michaelis-Mentennonlinear metabolic rate constants associated with HAART drug elimination

$\left(\sigma_{i}, \lambda_{i}\right)$ : Michaelis-Mentennonlinear metabolic rate constants associated with HAART drug pharmacokinetics $(i=2,3)$

$\xi_{I} \quad$ : Cytotoxic coefficient where $0 \leq \xi_{i} \leq 1(i=2,3)$

$q_{i}$ : Constant depicting competition between infected and un-infected CD4 ${ }^{+} \mathrm{T}_{4}$ helper cells $(i$ $=1,2)$

$k_{i} \quad$ : Constant depicting degradation, loss of clonogenicity or "death" $(i=1,2,3,4)$

$k_{5} \quad$ : Rate constant depicting exponential drug elimination pharmacokinetics

$e_{i 0}$ : Constant depicting death or degradation or removal by apoptosis (programmed cell death $)(i=1,2,3,4)$

$K_{i}$ : Constant associated with the killing rate of infected $\mathrm{CD}^{+} \mathrm{T}_{4}$ cells by $\mathrm{CD}^{+} \mathrm{T}_{8}$ cytotoxic lymphocytes $(i=1,2)$

All the parameters are positive.

\section{Model Equations}

\subsection{Description of the Model Equations}

The HIV-1 patho-physiological dynamics during HAART therapy can be modeled using the following system of non-linear ordinary differential equations. A detailed description of the dynamics of the model variables $x_{1}, x_{2}, x_{3}$, and $x_{4}$ can be found in Nani and Jin [15].

The model includes the following clinical improvements:

(i) The drug delivery uses transdermal, stealth-liposome encapsulated drug delivery, instead of the matrix tablet form because of improved therapeutic efficacy and reduced gastrointestinal toxicity [12]. It is also assumed that elastic liposomes are formulated and selectively targeted such as to reduce toxicity to non-HIV-1-infected CD4 ${ }^{+} \mathrm{T}$ cells $\left(x_{1}\right)$ and $\mathrm{CD}^{+}$cytotoxic $\mathrm{T}$ cells $\left(x_{4}\right)$.

(ii) The HAART drug is such that each renal excretion and body clearance rate follows Michaelis-Menten kinetics.

(iii) $g\left(x_{1}, x_{j}\right)=a_{j} x_{1} x_{j} e^{-b_{j} x_{1}}$ for $j=(1,2,4)$ 
This function depicts the process of lymphocyte activation which is mediated by $x_{1}$ $\left(\mathrm{CD}^{+}\right)$T helper cells. These cells secrete a cytokine called interleukin-2.

(iv) The periodic input function $f(t)=|\operatorname{ceil}(\sin (5 t))|$ can be depicted by the following plot:

$$
\begin{aligned}
& \int \dot{x}_{1}=S_{1}+a_{1} x_{1}^{2} e^{-b_{1} x_{1}}-\alpha_{1} x_{1} x_{3}-q_{1} x_{1} x_{2}-k_{1} x_{1}-e_{10} \\
& \dot{x}_{2}=S_{2}+a_{2} x_{1} x_{2} e^{-b_{2} x_{1}}+\alpha_{2} x_{1} x_{3}-q_{2} x_{1} x_{2}-k_{2} x_{2}-\beta_{1} x_{3} \\
& -K_{1} x_{2} x_{4}-e_{20}-\frac{\xi_{2} \sigma_{2} x_{2} x_{5}}{\lambda_{2}+x_{5}} \\
& \dot{x}_{3}=S_{3}+\beta_{2} x_{2} x_{3}+\beta_{3} x_{3}-\alpha_{3} x_{1} x_{3}-\eta_{1} x_{3} x_{4}-k_{3} x_{3}-e_{30} \\
& -\frac{\xi_{3} \sigma_{3} x_{3} x_{5}}{\lambda_{3}+x_{5}} \\
& \dot{x}_{4}=S_{4}+a_{4} x_{1} x_{4} e^{-b_{4} x_{1}}-K_{2} x_{2} x_{4}-\eta_{2} x_{3} x_{4}-k_{4} x_{4}-e_{40} \\
& \dot{x}_{5}=D f(t)-\frac{\sigma_{0} x_{5}}{\lambda_{0}+x_{5}}-\frac{\sigma_{2} x_{2} x_{5}}{\lambda_{2}+x_{5}}-\frac{\sigma_{3} x_{3} x_{5}}{\lambda_{3}+x_{5}}-k_{5} x_{5} \\
& \begin{array}{l}
f(t)=\left\{\begin{array}{cl}
1 & \text { for constant continuous input } \\
|\lceil\sin n t\rceil| & \text { for periodic input }
\end{array}\right. \\
x_{i}\left(t_{0}\right)=x_{i 0} \text { for }
\end{array}
\end{aligned}
$$

Transdermal Input Function $\mathrm{f}^{\prime}(\mathrm{t})$

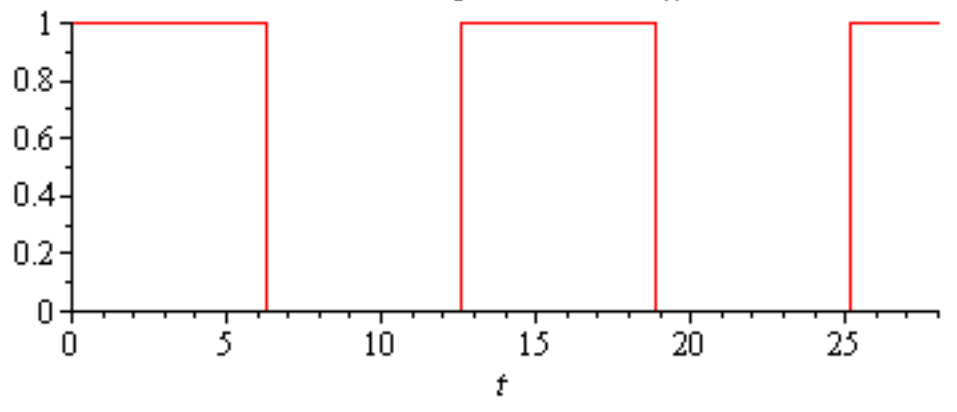

\subsection{Boundedness and Invariance of Non-negativity of Solutions}

In this subsection theoretical conditions will be constructed under which solutions to the HAART mathematical model equations are well-posed, ultimately bounded, and exhibit invariance of nonnegativity for all $t \in\left[t_{0}, T\right] \subset \Re_{+}=[0, \infty)$. In this case, $\mathrm{t}_{0}$, and $\mathrm{T}$ are defined respectively as times at which HAART therapy begins and terminates.

Theorem 3.1 Consider 
(i)

$$
\Omega=\left\{\left(x_{1}, x_{2}, x_{3}, x_{4}, x_{5}\right) \in \Re_{+}^{5} \mid 0 \leq x_{i} \leq \Phi_{i} i=1,2,3,4,5\right\}
$$

Let

(ii)

$$
\Phi_{i}=\max \left\{\left(x_{i 0}, \frac{S_{i}+C_{i}-e_{i 0}}{k_{i}}\right), i=1,2,3,4\right\}
$$

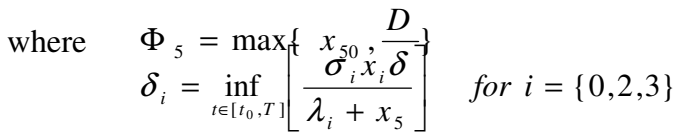

and

$\delta=\min \left\{\delta_{0}+\delta_{2}+\delta_{3}, k_{5}\right\}$,

Then there exists a $T_{0}>0$ such that for $T_{0}<t<\infty$, all solutions to the HAART model equations (3.1) with initial values $x_{i 0} \in \mathfrak{R}^{5}{ }_{+}=\left\{x_{i} \in \mathfrak{R} \mid x_{i} \geq 0, i=(1,2,3,4,5)\right\}$ are ultimately bounded, dissipative, and will eventually enter the non-negatively invariant region $\Omega$. In particular, the solutions are trapped in the region $\Omega$ for all $t>T_{0} \subset \Re_{+}$

Proof. Using the result from Nani and Jin in [7], let

$$
\begin{aligned}
& C_{j}=\sup _{t \in\left[t_{0}, T\right]}\left\lfloor a_{j} x_{1} x_{j} e^{-b_{j} x_{1}}\right\rfloor \text { for } j=\{1,2,4\} \\
& C_{3}=\sup _{t \in\left[t_{0}, T\right]}\left[\beta_{2} x_{2} x_{3}+\beta_{3} x_{3}\right]
\end{aligned}
$$

The non-linear system (4.1) can be converted to the following non-linear differential inequalities:

$$
\left\{\begin{array}{l}
\dot{x}_{1} \leq S_{1}+C_{1}-k_{1} x_{1}-e_{10} \\
\dot{x}_{2} \leq S_{2}+C_{2}-k_{2} x_{2}-e_{20} \\
\dot{x}_{3} \leq S_{3}+C_{3}-k_{3} x_{3}-e_{30} \\
\dot{x}_{4} \leq S_{4}+C_{4}-k_{4} x_{4}-e_{40} \\
\dot{x}_{5} \leq D-\delta x_{5} \\
x_{i}\left(t_{0}\right)=x_{i 0} \text { where } \quad i=\{1,2,3,4,5\}
\end{array}\right.
$$

Theorem [[Kamke: (ref. [29] Theorem. 2.6.3)]]

Consider the differential inequality

$$
\begin{aligned}
& \left\{\begin{array}{l}
\frac{d x_{i}}{d t} \leq f_{i}\left(x_{1}, x_{2}, \ldots x_{n}\right) \\
x_{i}\left(t_{0}\right)=x_{i 0}
\end{array} \text { with } f: C\left(R^{n}, R\right)\right. \\
& \text { where } t \in\left[t_{0}, b\right], b \in R_{+}=(0, \infty)
\end{aligned}
$$


Suppose

$$
\left\{\begin{array}{l}
\frac{d u_{i}}{d t}=f_{i}\left(u_{1}, u_{2}, \ldots u_{n}\right) \\
u_{i}\left(t_{0}\right)=u_{i 0}
\end{array}\right.
$$

such that $\quad x_{i 0} \leq u_{i 0}$

Then $\quad x_{i}(t) \leq u_{i}(t)$

Applying Kamke theorem to the system (3.4), the following inequalities hold.

$$
\left\{\begin{array}{l}
x_{i} \leq \frac{S_{i}+C_{i}-e_{i 0}}{k_{i}}+\gamma_{i} e^{-k_{i} t} \quad \text { for } i=\{1,2,3,4\}, \quad \text { where } S_{i}+C_{i}-e_{i 0}>0 \\
x_{5} \leq \frac{D}{\delta}+\gamma_{5} e^{-\delta t} \text { and } \gamma_{i} \in R_{+}=(0, \infty) \text { and } i=\{1,2,3,4,5\}
\end{array}\right.
$$

In particular, the following results can be obtained.

$$
\begin{aligned}
& \lim \sup x_{t}(t) \leq \frac{S_{i}+C_{i}-e_{i 0}}{k_{i}}, \quad i=\{1,2,3,4\} \\
& \lim \sup x_{5}(t) \leq \frac{D}{\delta}
\end{aligned}
$$

Thus, $\Omega$ is non-negatively invariant and the system is ultimately bounded, dissipative, with the bounds defined by the following equations.

$$
\begin{aligned}
& \sup _{t \in\left[t_{0}, T\right]} x_{i}(t)=\max _{t \in\left[t_{L}, t_{P}\right]}\left\{x_{i 0}, \frac{S_{i}+C_{i}-e_{i 0}}{k_{i}}\right\}, \quad i=\{1,2,3,4\} \\
& \sup _{t \in\left[t_{0}, T\right]} x_{5}(t)=\max _{t \in\left[t_{0}, T\right]}\left\{x_{50}, \frac{D}{\delta}\right\}
\end{aligned}
$$

As a consequence of (3.7), the orbits of $x_{i}(t)$ are uniformly bounded.

This completes the proof.

\section{The Equilibria and Computation of the Jacobian Matrices}

In this section, the model equations will be restricted to the case for which the drug input function has the form $f(t)=1$. Thus, (3.1) reduces to a system (4.1) of deterministic, non-linear, and autonomous ordinary differential equations. 


$$
\left\{\begin{array}{l}
\dot{x}_{1}=S_{1}+a_{1} x_{1}^{2} e^{-b_{1} x_{1}}-\alpha_{1} x_{1} x_{3}-q_{1} x_{1} x_{2}-k_{1} x_{1}-e_{10} \\
\dot{x}_{2}=S_{2}+a_{2} x_{1} x_{2} e^{-b_{2} x_{1}}+\alpha_{2} x_{1} x_{3}-q_{2} x_{1} x_{2}-k_{2} x_{2}-\beta_{1} x_{3}-K_{1} x_{2} x_{4}-e_{20}-\frac{\xi_{2} \sigma_{2} x_{2} x_{5}}{\lambda_{2}+x_{5}} \\
\dot{x}_{3}=S_{3}+\beta_{2} x_{2} x_{3}+\beta_{3} x_{3}-\alpha_{3} x_{1} x_{3}-\eta_{1} x_{3} x_{4}-k_{3} x_{3}-e_{30}-\frac{\xi_{3} \sigma_{3} x_{3} x_{5}}{\lambda_{3}+x_{5}} \\
\dot{x}_{4}=S_{4}+a_{4} x_{1} x_{4} e^{-b_{4} x_{1}}-K_{2} x_{2} x_{4}-\eta_{2} x_{3} x_{4}-k_{4} x_{4}-e_{40} \\
\dot{x}_{5}=D-\frac{\sigma_{0} x_{5}}{\lambda_{0}+x_{5}}-\frac{\sigma_{2} x_{2} x_{5}}{\lambda_{2}+x_{5}}-\frac{\sigma_{3} x_{3} x_{5}}{\lambda_{3}+x_{5}}-k_{5} x_{5} \\
x_{i}\left(t_{0}\right)=x_{i 0} \text { for } \quad i=\{1,2,3,4,5\}
\end{array}\right.
$$

The physiological outcomes or the equilibria during constant continuous transdermal HAART therapy occur when $\dot{x}_{i}=0$ for $i=1,2,3,4,5$.

\subsection{Local Existence Criteria for Clinically Relevant Steady States of HAART}

In this subsection, a subset of clinically relevant states or the possible patho-physiological outcomes from constant continuous transdermal HAART therapy are listed and analyzed. These outcomes are labelled $E_{i}$ for $i=1,2, \ldots, 10$. The local existence criteria are labelled $C_{i}$.

(i) $\quad E_{1}=\left[0,0,0,0, x_{5}\right]$

$$
C_{1}:\left\{\begin{array}{l}
S_{i}-e_{i 0}=0 \quad i=\{1,2,3,4\} \\
D-\frac{\sigma_{0} x_{5}}{\lambda_{0}+x_{5}}-k_{5} x_{5}=0 \quad \text { for } \quad x_{5} \in \Omega
\end{array}\right.
$$

This equilibrium represents the scenario in which HAART annihilates the HIV-1 virions, the noninfected CD4+, the infected CD4+, and CD8+ T cells. The immuno-compromised patient may become incapacitated with opportunistic infections.

(ii) $E_{2}=\left[0,0,0, x_{4}, x_{5}\right]$

$$
C_{2}:\left\{\begin{array}{l}
S_{i}-e_{i 0}=0 \quad i=\{1,2,3\} \\
D-\frac{\sigma_{0} x_{5}}{\lambda_{0}+x_{5}}-k_{5} x_{5}=0 \quad \text { for } \quad x_{5} \in \Omega
\end{array}\right.
$$

This equilibrium is similar to case (i) except that HIV-1 specific CD8+ cells are now present in the steady state.

(iii) $\quad E_{3}=\left[x_{1}, 0,0, x_{4}, x_{5}\right]$

$$
C_{3}:\left\{\begin{array}{l}
S_{1}+a_{1} x_{1}^{2} e^{-b_{1} x_{1}}-k_{1} x_{1}-e_{10}=0 \\
S_{i}-e_{i 0}=0 \quad i=\{2,3\} \\
S_{4}+a_{4} x_{1} x_{4} e^{-b_{4} x_{1}}-k_{4} x_{4}-e_{40}=0 \\
D-\frac{\sigma_{0} x_{5}}{\lambda_{0}+x_{5}}-k_{5} x_{5}=0 \quad \text { for } x_{1}, x_{4}, x_{5} \in \Omega
\end{array}\right.
$$


This equilibrium corresponds to a scenario of successful HAART protocol in which the steady state consists of some uninfected CD4+ T cells, HIV-1 specific CD8+ T cells, and some drug residue.

(iv) $E_{4}=\left[x_{1}, 0,0,0, x_{5}\right]$

$$
C_{4}:\left\{\begin{array}{l}
S_{1}+a_{1} x_{1}^{2} e^{-b_{1} x_{1}}-k_{1} x_{1}-e_{10}=0 \\
S_{i}-e_{i 0}=0 \quad i=\{2,3,4\} \\
D-\frac{\sigma_{0} x_{5}}{\lambda_{0}+x_{5}}-k_{5} x_{5}=0 \quad \text { for } x_{1}, x_{5} \in \Omega
\end{array}\right.
$$

This equilibrium also corresponds to a scenario of successful HAART protocol in which the steady state consists of some uninfected CD4+ T cells and some drug residue but no HIV-1 specific CD8+ T cells.

(v) $E_{5}=\left[0, x_{2}, x_{3}, 0, x_{5}\right]$

$$
\mathrm{C}_{5}:\left\{\begin{array}{l}
S_{i}-e_{i 0}=0 \quad i=\{1,4\} \\
S_{2}-k_{2} x_{2}-\beta_{1} x_{3}-e_{20}-\frac{\xi_{2} \sigma_{2} x_{2} x_{5}}{\lambda_{2}+x_{5}}=0 \\
S_{3}+\beta_{2} x_{2} x_{3}+\beta_{3} x_{3}-k_{3} x_{3}-e_{30}-\frac{\xi_{3} \sigma_{3} x_{3} x_{5}}{\lambda_{3}+x_{5}}=0 \\
D-\frac{\sigma_{0} x_{5}}{\lambda_{0}+x_{5}}-\frac{\sigma_{2} x_{2} x_{5}}{\lambda_{2}+x_{5}}-\frac{\sigma_{3} x_{3} x_{5}}{\lambda_{3}+x_{5}}-k_{5} x_{5}=0 \quad \text { for } x_{2}, x_{3}, x_{5} \in \Omega
\end{array}\right.
$$

This equilibrium exhibits a failure of HAART protocol. In particular, the HIV-1 infected CD4+ T cells and HIV-1 virions populate the steady state in addition to some drug residue whereas the non-infected CD4+ and CD8+ T cells are annihilated.

(vi) $E_{6}=\left[0,0, x_{3}, 0, x_{5}\right]$

$$
\mathrm{C}_{6}:\left\{\begin{array}{l}
S_{i}-e_{i 0}=0 \quad i=\{1,2,4\} \\
S_{3}+\beta_{3} x_{3}-k_{3} x_{3}-e_{30}-\frac{\xi_{3} \sigma_{3} x_{3} x_{5}}{\lambda_{3}+x_{5}}=0 \\
D-\frac{\sigma_{0} x_{5}}{\lambda_{0}+x_{5}}-\frac{\sigma_{3} x_{3} x_{5}}{\lambda_{3}+x_{5}}-k_{5} x_{5}=0 \quad \text { for } \quad x_{3}, x_{5} \in \Omega
\end{array}\right.
$$

This equilibrium also depicts a case of therapeutic failure of HAART because HIV-1 virions still remain in the blood plasma of the patient.

$$
\begin{aligned}
& \text { (vii) } E_{7}=\left[0, x_{2}, 0,0, x_{5}\right] \\
& \mathrm{C}_{7}:\left\{\begin{array}{l}
S_{i}-e_{i 0}=0 \quad i=\{1,3,4\} \\
S_{2}-k_{2} x_{2}-e_{20}-\frac{\xi_{2} \sigma_{2} x_{2} x_{5}}{\lambda_{2}+x_{5}}=0 \\
D-\frac{\sigma_{0} x_{5}}{\lambda_{0}+x_{5}}-\frac{\sigma_{2} x_{2} x_{5}}{\lambda_{2}+x_{5}}-k_{5} x_{5}=0 \quad \text { for } x_{2}, x_{5} \in \Omega
\end{array}\right.
\end{aligned}
$$


This equilibrium represents another a scenario of unsuccessful HAART protocol because HIV-1 infected CD4+ T cells and drug residue populate the steady state.

$$
\begin{aligned}
& \text { (viii) } E_{8}=\left[x_{1}, x_{2}, 0,0, x_{5}\right] \\
& \mathrm{C}_{8}:\left\{\begin{array}{l}
S_{i}-e_{i 0}=0 \quad i=\{3,4\} \\
S_{1}+a_{1} x_{1}^{2} e^{-b_{1} x_{1}}-k_{1} x_{1}-q_{1} x_{1} x_{2}-e_{10}=0 \\
S_{2}+a_{2} x_{1} x_{2} e^{-b_{2} x_{1}}-q_{2} x_{1} x_{2}-k_{2} x_{2}-e_{20}-\frac{\xi_{2} \sigma_{2} x_{2} x_{5}}{\lambda_{2}+x_{5}}=0 \\
D-\frac{\sigma_{0} x_{5}}{\lambda_{0}+x_{5}}-\frac{\sigma_{2} x_{2} x_{5}}{\lambda_{2}+x_{5}}-k_{5} x_{5}=0 \quad \text { for } \quad x_{1}, x_{2}, x_{5} \in \Omega
\end{array}\right.
\end{aligned}
$$

This equilibrium is not clinically desirable because of existence of HIV-1 infected CD4+ T cells.

$$
\begin{aligned}
& \text { (ix) } E_{9}=\left[x_{1}, x_{2}, 0, x_{4}, x_{5}\right] \\
& \mathrm{C}_{9}:\left\{\begin{array}{l}
S_{1}+a_{1} x_{1}^{2} e^{-b_{1} x_{1}}-q_{1} x_{1} x_{2}-k_{1} x_{1}-e_{10}=0 \\
S_{2}+a_{2} x_{1} x_{2} e^{-b_{2} x_{1}}-q_{2} x_{1} x_{2}-k_{2} x_{2}-K_{1} x_{2} x_{4}-e_{20}-\frac{\xi_{2} \sigma_{2} x_{2} x_{5}}{\lambda_{2}+x_{5}}=0 \\
S_{3}-e_{30}=0 \\
S_{4}+a_{4} x_{1} x_{4} e^{-b_{4} x_{1}}-K_{2} x_{2} x_{4}-k_{4} x_{4}-e_{40}=0 \\
D-\frac{\sigma_{0} x_{5}}{\lambda_{0}+x_{5}}-\frac{\sigma_{2} x_{2} x_{5}}{\lambda_{2}+x_{5}}-k_{5} x_{5} \quad \text { for } \quad x_{1}, x_{2}, x_{3}, x_{5} \in \Omega
\end{array}\right.
\end{aligned}
$$

This equilibrium is another scenario of therapeutic failure of HAART protocol because HIV-1 infected CD4+ T cells are all present.

$$
\text { (x) } \quad E_{10}=\left[x_{1}, x_{2}, x_{3}, x_{4}, x_{5}\right]
$$

The steady state $E_{10}$ depicts a person who is living with AIDS. In this case, the model exhibits persistence and the viral titer is not sufficient to annihilate the immune system. The criteria for persistence of HIV-1 AIDS during HAART will be discussed in Theorem 5.5.

There are some other steady states which are not listed because they are less clinically interesting.

\subsection{Computation of the Jacobian Matrices}

Using the Hartman-Grobman theorem, it is possible to investigate the therapeutic efficacy of HAART and the physiological stability of HIV-1 AIDS disease dynamics associated with the model equations, by linearizing the system (4.1) in the neighborhood of the equilibria.

The Jacobian matrix of linearization near any physiological outcome is denoted symbolically by

$$
J\left[E_{k}\right]:=\left\{a_{i j}\right\}_{5 \times 5} \in M_{5 \times 5}(\Re) \quad k=3,4,5
$$


In particular, the $a_{i j}$ entries are defined as follows:

$$
\begin{aligned}
& a_{11}:=a_{1} x_{1}\left(2-b_{1} x_{1}\right) e^{-b_{1} x_{1}}-\alpha_{1} x_{3}-q_{1} x_{2}-k_{1} \\
& a_{12}:=-q_{1} x_{1} \\
& a_{13}:=-\alpha_{1} x_{1} \\
& a_{14}:=0 \\
& a_{15}:=0 \\
& a_{21}:=a_{2} x_{2}\left(1-b_{2} x_{1}\right) e^{-b_{2} x_{1}}-q_{2} x_{2} \\
& a_{22}:=a_{2} x_{1} e^{-b_{2} x_{1}}-q_{2} x_{1}-k_{2}-K_{1} x_{4}-\frac{\xi_{2} \sigma_{2} x_{5}}{\lambda_{2}+x_{5}} \\
& a_{23}:=\alpha_{2} x_{1}-\beta_{1} \\
& a_{24}:=-K_{1} x_{2} \\
& a_{25}:=-\frac{\xi_{2} \lambda_{2} \sigma_{2} x_{2}}{\left(\lambda_{2}+x_{5}\right)^{2}} \\
& \text { (4.2) } \quad a_{31}:=-\alpha_{3} x_{3} \\
& a_{32}:=\beta_{2} x_{3} \\
& a_{33}:=\beta_{2} x_{2}+\beta_{3}-\alpha_{3} x_{1}-\eta_{1} x_{4}-k_{3}-\frac{\xi_{3} \sigma_{3} x_{5}}{\lambda_{3}+x_{5}} \\
& a_{34}:=-\eta_{1} x_{3} \\
& a_{35}:=-\frac{\xi_{3} \sigma_{3} \lambda_{3}}{\left(\lambda_{3}+x_{5}\right)^{2}} \\
& a_{41}:=a_{4} x_{4}\left(1-b_{4} x_{1}\right) e^{-b_{4} x_{1}} \\
& a_{42}:=-K_{2} x_{4} \\
& a_{43}:=-\eta_{2} x_{4} \\
& a_{44}:=a_{4} x_{1} e^{-b_{4} x_{1}}-K_{2} x_{2}-\eta_{2} x_{3}-k_{4} \\
& a_{45}:=0 \\
& a_{51}:=0 \\
& a_{52}:=-\frac{\sigma_{2} x_{5}}{\lambda_{2}+x_{5}} \\
& a_{53}:=-\frac{\sigma_{3} x_{5}}{\lambda_{3}+x_{5}} \\
& a_{54}:=0 \\
& a_{55}:=-\frac{\sigma_{0} \lambda_{0}}{\left(\lambda_{0}+x_{5}\right)^{2}}-\frac{\sigma_{2} \lambda_{2} x_{2}}{\left(\lambda_{2}+x_{5}\right)^{2}}-\frac{\sigma_{3} \lambda_{3} x_{3}}{\left(\lambda_{3}+x_{5}\right)^{2}}-k_{5}
\end{aligned}
$$

The Jacobian matrices for the steady states $E_{3}, E_{4}, E_{5}$ are respectively listed as follows: 


$$
\begin{aligned}
& J\left\{E_{1}\left[0,0,0,0, x_{5}\right]\right\}= \\
& {\left[\begin{array}{ccccc}
-k_{1} & 0 & 0 & 0 & 0 \\
0 & -k_{2}-\frac{\xi_{2} \sigma_{2} x_{5}}{\lambda_{2}+x_{5}} & -\beta_{1} & 0 & 0 \\
0 & 0 & \beta_{3}-k_{3}-\frac{\xi_{3} \sigma_{3} x_{5}}{\lambda_{3}+x_{5}} & 0 & -\frac{\xi_{3} \sigma_{3} \lambda_{3}}{\left(\lambda_{3}+x_{5}\right)^{2}} \\
0 & 0 & 0 & -k_{4} & 0 \\
0 & -\frac{\sigma_{2} x_{5}}{\lambda_{2}+x_{5}} & -\frac{\sigma_{3} x_{5}}{\lambda_{3}+x_{5}} & 0 & -\frac{\sigma_{0} \lambda_{0}}{\left(\lambda_{0}+x_{5}\right)^{2}}-k_{5}
\end{array}\right]}
\end{aligned}
$$

$$
J\left\{E_{2}\left[0,0,0, x_{4}, x_{5}\right]\right\}=
$$

$$
\left[\begin{array}{ccccc}
-k_{1} & 0 & 0 & 0 & 0 \\
0 & -k_{2}-K_{1} x_{4}-\frac{\xi_{2} \sigma_{2} x_{5}}{\lambda_{2}+x_{5}} & -\beta_{1} & 0 & 0 \\
0 & 0 & \beta_{3}-\eta_{1} x_{4}-k_{3}-\frac{\xi_{3} \sigma_{3} x_{5}}{\lambda_{3}+x_{5}} & 0 & -\frac{\xi_{3} \sigma_{3} \lambda_{3}}{\left(\lambda_{3}+x_{5}\right)^{2}} \\
a_{4} x_{4} & -K_{2} x_{4} & -\eta_{2} x_{4} & -k_{4} & 0 \\
0 & -\frac{\sigma_{2} x_{5}}{\lambda_{2}+x_{5}} & -\frac{\sigma_{3} x_{5}}{\lambda_{3}+x_{5}} & 0 & -\frac{\sigma_{0} \lambda_{0}}{\left(\lambda_{0}+x_{5}\right)^{2}}-k_{5}
\end{array}\right]
$$

$J\left\{E_{3}\left[x_{1}, 0,0, x_{4}, x_{5}\right]\right\}=$

$$
\left[\begin{array}{ccccc}
a_{1} x_{1}\left(2-b_{1} x_{1}\right) e^{-h_{4} x_{1}}-k_{1} & -q_{1} x_{1} & -\alpha_{1} x_{1} & 0 & 0 \\
0 & a_{2} x_{1} e^{-b_{2} x_{1}}-q_{2} x_{1}-k_{2}-K_{1} x_{4}-\frac{\xi_{2} \sigma_{2} x_{5}}{\lambda_{2}+x_{5}} & \alpha_{2} x_{1}-\beta_{1} & 0 & 0 \\
0 & 0 & \beta_{3}-\alpha_{3} x_{1}-\eta_{1} x_{4}-k_{3}-\frac{\xi_{3} \sigma_{3} x_{5}}{\lambda_{3}+x_{5}} & 0 & \frac{\xi_{3} \sigma_{3} \lambda_{3}}{\left(\lambda_{3}+x_{5}\right)^{2}} \\
a_{4} x_{4}\left(1-b_{4} x_{1}\right) e^{-b_{4} x_{1}} & -K_{2} x_{4} & -\eta_{2} x_{4} & a_{4} x_{1} e^{-b_{4} x_{4}}-k_{4} & 0 \\
0 & -\frac{\sigma_{2} x_{5}}{\lambda_{2}+x_{5}} & -\frac{\sigma_{3} x_{5}}{\lambda_{3}+x_{5}} & 0 & -\frac{\sigma_{0} \lambda_{5}}{\left(\lambda_{0}+x_{5}\right)^{2}}-k_{5}
\end{array}\right]
$$

$J\left\{E_{4}\left[x_{1}, 0,0,0, x_{5}\right]\right\}=$

$$
\left[\begin{array}{ccccc}
a_{1} x_{1}\left(2-b_{1} x_{1}\right) e^{-b_{1} x_{1}}-k_{1} & -q_{1} x_{1} & -\alpha_{1} x_{1} & 0 & 0 \\
0 & a_{2} x_{1} e^{-b_{2} x_{1}}-q_{2} x_{1}-k_{2}-\frac{\xi_{2} \sigma_{2} x_{5}}{\lambda_{2}+x_{5}} & \alpha_{2} x_{1}-\beta_{1} & 0 & 0 \\
0 & 0 & \beta_{3}-\alpha_{3} x_{1}-k_{3}-\frac{\xi_{3} \sigma_{3} x_{5}}{\lambda_{3}+x_{5}} & 0 & -\frac{\xi_{3} \sigma_{3} \lambda_{3}}{\left(\lambda_{3}+x_{5}\right)^{2}} \\
0 & 0 & 0 & a_{4} x_{1} e^{-b_{4} x_{1}}-k_{4} & 0 \\
0 & -\frac{\sigma_{2} x_{5}}{\lambda_{2}+x_{5}} & -\frac{\sigma_{3} x_{5}}{\lambda_{3}+x_{5}} & 0 & -\frac{\sigma_{0} \lambda_{0}}{\left(\lambda_{0}+x_{5}\right)^{2}}-k_{5}
\end{array}\right]
$$




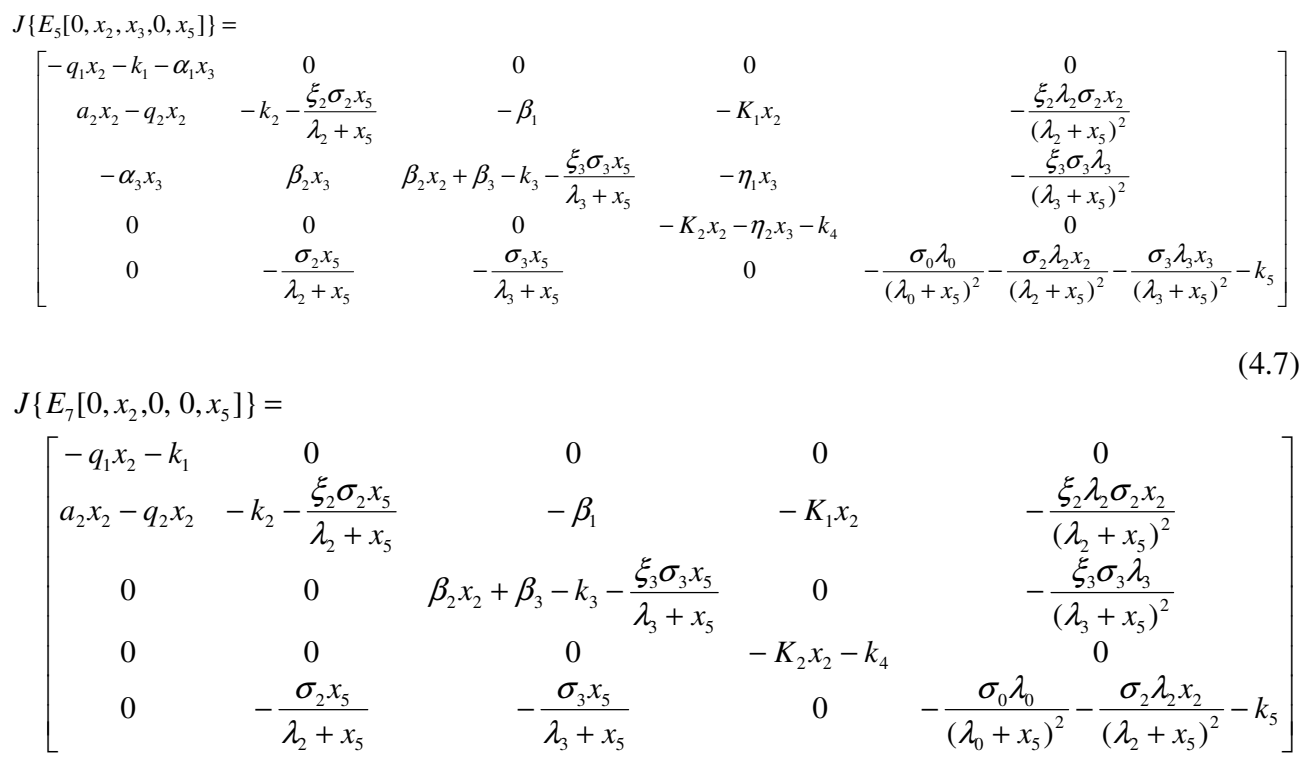

$J\left\{E_{9}\left[x_{1}, x_{2}, 0, x_{4}, x_{5}\right]\right\}=$

$\left[\begin{array}{ccccc}a_{1} x_{1}\left(2-b_{1} x_{1}\right) e^{-b_{1} x_{1}}+q_{1} x_{2}-k_{1} & -q_{1} x_{1} & -\alpha_{1} x_{1} & 0 & 0 \\ a_{2} x_{2}\left(1-b_{2} x_{1}\right) e^{-b_{2} x_{1}}+q_{2} x_{2} & a_{2} x_{1} e^{-b_{2} x_{1}}-q_{2} x_{1}-k_{2}-K_{1} x_{4}-\frac{\xi_{2} \sigma_{2} x_{5}}{\lambda_{2}+x_{5}} & \alpha_{2} x_{1}-\beta_{1} & -K_{1} x_{2} & -\frac{\xi_{2} \sigma_{2} \lambda_{2} x_{2}}{\left(\lambda_{2}+x_{5}\right)^{2}} \\ 0 & 0 & \beta_{2} x_{2}+\beta_{3}-\alpha_{3} x_{1} & & -\frac{\xi_{3} \sigma_{3} \lambda_{3}}{\left(\lambda_{3}+x_{5}\right)^{2}} \\ a_{4} x_{4}\left(1-b_{4} x_{1}\right) e^{-b_{4} x_{1}} & -\eta_{1} x_{4}-k_{3}-\frac{\xi_{3} \sigma_{3} x_{5}}{\lambda_{3}+x_{5}} & 0 & 0 \\ 0 & -\frac{\sigma_{2} x_{5}}{\lambda_{2}+x_{5}} & -\eta_{2} x_{4} & a_{4} x_{1} e^{-b_{4} x_{1}}+K_{2} x_{2}-k_{4} & \sigma_{3} x_{5} \\ \lambda_{3}+x_{5} & 0 & -\frac{\sigma_{0} \lambda_{0}}{\left(\lambda_{0}+x_{5}\right)^{2}}-\frac{\sigma_{2} \lambda_{2} x_{2}}{\left(\lambda_{2}+x_{5}\right)^{2}}-k_{5}\end{array}\right]$

\section{Necessary Criteria for Local Stability of Equilibria during HAART}

Let $E=\left\{E_{1}, E_{2}, \ldots, E_{10}\right\}$ denote the set of some clinically relevant equilibria of model equations (4.1). In this section, the local stability of $E_{4}, E_{5}, E_{1}$, and $E_{3}$ will be analyzed using the principles of linearized stability of autonomous ordinary differential equations. In particular, the necessary mathematical criteria for certain therapeutic outcomes during HAART are computed and presented in the form of theorems.

\subsection{Local Stability of $E_{4}=\left[\hat{x}_{1}, 0,0,0, \hat{x}_{5}\right]$}

The Jacobian matrix associated with $E_{4}=\left[\hat{x}_{1}, 0,0,0, \hat{x}_{5}\right]$ is defined as 


$$
J\left\{E_{4}\left[\hat{x}_{1}, 0,0,0, \hat{x}_{5}\right]\right\}:=\left|\begin{array}{ccccc}
\hat{a}_{11} & \hat{a}_{12} & \hat{a}_{13} & 0 & 0 \\
0 & \hat{a}_{22} & \hat{a}_{23} & 0 & 0 \\
0 & 0 & \hat{a}_{33} & 0 & \hat{a}_{35} \\
0 & 0 & 0 & \hat{a}_{44} & 0 \\
0 & \hat{a}_{52} & \hat{a}_{53} & 0 & \hat{a}_{55}
\end{array}\right|
$$

If the condition $\hat{x}_{1}=\frac{\beta_{1}}{\alpha_{2}}$ is imposed on the Jacobian matrix above, then $\hat{a}_{23}=0$. The eigenspectrum associated with the Jacobian matrix is defined as

$\sigma\left(J\left\{E_{4}\left[\hat{x}_{1}, 0,0,0, \hat{x}_{5}\right]\right\}\right)=\left\{\lambda_{i} \mid \lambda^{2}+\hat{a}_{1} \lambda+\hat{a}_{2}=0, i=1,2\right\} \cup\left\{\lambda_{3}, \lambda_{4}, \lambda_{5}\right\}$

where

$$
\left\{\begin{array}{l}
\hat{a}_{1}=-\left(\hat{a}_{33}+\hat{a}_{55}\right) \\
\hat{a}_{2}=\hat{a}_{33} \hat{a}_{55}-\hat{a}_{35} \hat{a}_{53} \\
\lambda_{3}=\hat{a}_{11} \\
\lambda_{4}=\hat{a}_{22} \\
\lambda_{5}=\hat{a}_{44}
\end{array}\right.
$$

Theorem 5.1. Suppose $C_{4}$ in Section 4.1 holds, and:

(i) $\left\{\begin{array}{l}\hat{a}_{1}>0 \\ \hat{a}_{2}>0 \\ \hat{x}_{1}=\frac{\beta_{1}}{\alpha_{2}}\end{array}\right.$
(ii) $\left\{\begin{array}{l}\hat{a}_{11}=a_{1} \hat{x}_{1}\left(2-b_{1} \hat{x}_{1}\right) e^{-b_{1} \hat{x}_{1}}-k_{1}<0 \\ \hat{a}_{22}=a_{2} \hat{x}_{1} e^{-b_{2} \hat{x}_{1}}-q_{2} \hat{x}_{1}-k_{2}-\frac{\xi_{2} \sigma_{2} \hat{x}_{5}}{\lambda_{2}+\hat{x}_{5}}<0 \\ \hat{a}_{44}=a_{4} \hat{x}_{1} e^{-b_{4} \hat{x}_{1}}-k_{4}<0\end{array}\right.$

Then the HAART therapeutic outcome $E_{4}=\left[\hat{x}_{1}, 0,0,0, \hat{x}_{5}\right]$ exists and it is a local attractor.

Proof. Theeigen-values of $J\left\{E_{4}\left[\hat{x}_{1}, 0,0,0, \hat{x}_{5}\right]\right\}$ satisfy the determinantal equation: 


$$
\begin{aligned}
& \operatorname{det}\left|J\left\{E_{4}\left[\hat{x}_{1}, 0,0,0, \hat{x}_{5}\right]\right\}-\lambda I\right|= \\
& \operatorname{det}\left|\begin{array}{ccccc}
\hat{a}_{11}-\lambda & \hat{a}_{12} & \hat{a}_{13} & 0 & 0 \\
0 & \hat{a}_{22}-\lambda & \hat{a}_{23} & 0 & 0 \\
0 & 0 & \hat{a}_{33}-\lambda & 0 & \hat{a}_{35} \\
0 & 0 & 0 & \hat{a}_{44}-\lambda & 0 \\
0 & \hat{a}_{52} & \hat{a}_{53} & 0 & \hat{a}_{55}-\lambda
\end{array}\right|=0
\end{aligned}
$$

Using the Laplace expansion technique for computation of determinant, the characteristic eigenvalue polynomial is given by

$$
p\left(\lambda, J\left(E_{4}\right)\right)=\left(\hat{a}_{11}-\lambda\right)\left(\hat{a}_{22}-\lambda\right)\left(\hat{a}_{44}-\lambda\right) \operatorname{det}\left|\begin{array}{cc}
\hat{a}_{33}-\lambda & \hat{a}_{35} \\
\hat{a}_{53} & \hat{a}_{55}-\lambda
\end{array}\right|=0
$$

But

$$
\operatorname{det}\left|\begin{array}{cc}
\hat{a}_{33}-\lambda & \hat{a}_{35} \\
\hat{a}_{53} & \hat{a}_{55}-\lambda
\end{array}\right|:=\lambda^{2}-\left(\hat{a}_{33}+\hat{a}_{55}\right) \lambda+\operatorname{det}\left|\begin{array}{cc}
\hat{a}_{33} & \hat{a}_{35} \\
\hat{a}_{53} & \hat{a}_{55}
\end{array}\right|
$$

Let

$$
\begin{aligned}
& \hat{a}_{1}=-\left(\hat{a}_{33}+\hat{a}_{55}\right) \\
& \hat{a}_{2}=\operatorname{det}\left|\begin{array}{ll}
\hat{a}_{33} & \hat{a}_{35} \\
\hat{a}_{53} & \hat{a}_{55}
\end{array}\right|
\end{aligned}
$$

The eigen-spectrum is therefore given by

$$
\sigma\left(J\left\{E_{4}\left[\hat{x}_{1}, 0,0,0, \hat{x}_{5}\right]\right\}\right)=\left\{\lambda_{i} \mid \lambda^{2}+\hat{a}_{1} \lambda+\hat{a}_{2}=0, i=1,2\right\} \cup\left\{\lambda_{3}, \lambda_{4}, \lambda_{5}\right\}
$$

where

$$
\begin{aligned}
& \lambda_{1}=\frac{1}{2}\left(-\hat{a}_{1}-\sqrt{\hat{a}_{1}^{2}-4 \hat{a}_{2}}\right) \\
& \lambda_{2}=\frac{1}{2}\left(-\hat{a}_{1}+\sqrt{\hat{a}_{1}^{2}-4 \hat{a}_{2}}\right) \\
& \lambda_{3}=\hat{a}_{11} \\
& \lambda_{4}=\hat{a}_{22} \\
& \lambda_{5}=\hat{a}_{44}
\end{aligned}
$$

The conditions (5.1) and (5.2) guarantee that the eigen-values associated with the equilibrium E4 have negative real part by virtue of Routh-Hurwicz criteria [7]. Hence, the equilibrium is locally asymptotically stable and a local attractor as well as a hyperbolic sink. This theorem establishes 
the local existence and local stability of a clinically desirable physiological outcome of HAART protocol.

Clinical Implication 5.1. The criteria (5.1) and (5.2) guarantee a temporary cure for the AIDS patient. There will be a finite time interval during which the HIV-1 virions will be annihilated from the patient's blood plasma. This will however be short-lived because the rest point $E_{4}\left[x_{1}, 0,0\right.$, $\left.0, x_{5}\right]$ may become unstable and the criteria for temporal cure are violated. It is possible for therapeutic criteria to be derived to maintain the patient to be permanently free of AIDS, which we shall discuss in Theorem 5.5.

\subsection{Local Stability of $E_{5}=\left[0, \breve{x}_{2}, \breve{x}_{3}, 0, \breve{x}_{5}\right]$}

In this subsection, the local existence and stability of $E_{5}=\left[0, \breve{x}_{2}, \breve{x}_{3}, 0, \breve{x}_{5}\right]$ will be analyzed. The results are summarized in the following theorem.

\section{Theorem 5.2. Suppose}

(i) Existence criteria $C_{5}$ for equilibrium $E_{5}=\left[0, \breve{x}_{2}, \breve{x}_{3}, 0, \breve{x}_{5}\right]$ holds.

(ii) Let $\sigma\left(J\left\{E_{5}\left[0, \breve{x}_{2}, \breve{x}_{3}, 0, \breve{x}_{5}\right]\right\}\right)$ be the eigen-spectrum of

$$
J\left\{E_{5}\left[0, \breve{x}_{2}, \breve{x}_{3}, 0, \breve{x}_{5}\right]\right\}:=\left|\begin{array}{ccccc}
\breve{a}_{11} & 0 & 0 & 0 & 0 \\
\breve{a}_{21} & \bar{a}_{22} & \breve{a}_{23} & \breve{a}_{24} & \breve{a}_{25} \\
\breve{a}_{31} & \breve{a}_{32} & \breve{a}_{33} & \breve{a}_{34} & \breve{a}_{35} \\
0 & 0 & 0 & \breve{a}_{44} & 0 \\
0 & \breve{a}_{52} & \breve{a}_{53} & 0 & \breve{a}_{55}
\end{array}\right|
$$

with the $\breve{a}_{i j}$ are depicted by (4.7).

such that

$$
\sigma\left(J\left\{E_{5}\left[0, \breve{x}_{2}, \breve{x}_{3}, 0, \breve{x}_{5}\right]\right\}\right)=\left\{\lambda_{i} \mid \lambda^{3}+\breve{a}_{1} \lambda^{2}+\breve{a}_{2} \lambda+\breve{a}_{3}=0, i=1,2,3\right\} \cup\left\{\lambda_{4}, \lambda_{5}\right\}
$$

Where

$$
\begin{aligned}
& \breve{a}_{1}=-\operatorname{Trace}\left[\begin{array}{lll}
a_{22} & a_{23} & a_{25} \\
a_{32} & a_{33} & a_{35} \\
a_{52} & a_{53} & a_{55}
\end{array}\right] \\
& \breve{a}_{2}=\operatorname{det}\left|\begin{array}{ll}
\breve{a}_{33} & \breve{a}_{35} \\
\breve{a}_{53} & \breve{a}_{55}
\end{array}\right|+\operatorname{det}\left|\begin{array}{ll}
\breve{a}_{22} & \breve{a}_{25} \\
\breve{a}_{31} & \breve{a}_{33}
\end{array}\right|+\operatorname{det}\left|\begin{array}{ll}
\breve{a}_{22} & \breve{a}_{23} \\
\breve{a}_{32} & \breve{a}_{33}
\end{array}\right| \\
& \breve{a}_{3}=-\operatorname{det}\left|\begin{array}{lll}
\breve{a}_{22} & \breve{a}_{23} & \breve{a}_{25} \\
\breve{a}_{32} & \breve{a}_{33} & \breve{a}_{35} \\
\breve{a}_{52} & \breve{a}_{53} & \breve{a}_{55}
\end{array}\right| \\
& \lambda_{4}=\breve{a}_{11} \\
& \lambda_{5}=\breve{a}_{44}
\end{aligned}
$$


Then the rest point $E_{5}\left[0, \breve{x}_{2}, \breve{x}_{3}, 0, \breve{x}_{5}\right]$ is locally asymptotically stable hyperbolic sink and a local attractor if

$$
\breve{a}_{1}>0, \breve{a}_{3}>0, \quad \breve{a}_{1} \breve{a}_{2}>\breve{a}_{3}
$$

Proof. The eigen-values associated with $E_{5}\left[0, \breve{x}_{2}, \breve{x}_{3}, 0, \breve{x}_{5}\right]$ satisfy

$$
\operatorname{det}\left|J\left\{E_{5}\left[0, \breve{x}_{2}, \breve{x}_{3}, 0, \breve{x}_{5}\right]\right\}-\lambda I_{5}\right|=0
$$

Using the Laplace expansion technique of determinants leads to (5.6) with the Routh-Hurwicz coefficients depicted by (5.7). The conditions specified in (5.8) guarantee that the equilibrium is locally asymptotically stable and a local attractor.

Clinical Implication 5.2. This theorem depicts the conditions for one of the worst scenarios during AIDS therapy using continuous infusion HAART. If the conditions (5.4) and (5.8) hold, then the HAART therapy annihilated all the uninfected $\mathrm{CD}^{+} \mathrm{T}$ cells, and HIV-1 specific cytotoxic $\mathrm{CD}^{+} \mathrm{T}$ cells. Consequently, the immune system of the AIDS patient becomes incapacitated and the patient becomes the target of opportunistic infections. However, this situation may not last very long but could be fatal.

\subsection{Local Stability of $E_{1}=\left[0,0,0,0, \tilde{x}_{5}\right]$}

In this subsection, the local existence and local stability of the non-clinically efficacious equilibrium $E_{1}$ will be discussed. Theoretical criteria under which $E_{1}=\left[0,0,0,0, \tilde{x}_{5}\right]$ is locally stable will be derived. The results are summarized in the following theorem.

Theorem 5.3. Suppose

(i) The criterion $C_{5}$ holds for the local existence of $E_{1}=\left[0,0,0,0, \tilde{x}_{5}\right]$

(ii) Let

$$
\tilde{M}=\left[\begin{array}{ccc}
\tilde{a}_{22} & \tilde{a}_{23} & 0 \\
0 & \tilde{a}_{33} & \tilde{a}_{35} \\
\tilde{a}_{52} & \tilde{a}_{53} & \tilde{a}_{55}
\end{array}\right]
$$

Such that

$$
\begin{aligned}
& \tilde{a}_{1}=-\operatorname{Trace}(\tilde{M}) \\
& \tilde{a}_{2}=\operatorname{det}\left|\begin{array}{ll}
\tilde{a}_{33} & \tilde{a}_{35} \\
\tilde{a}_{53} & \tilde{a}_{55}
\end{array}\right|+\tilde{a}_{22}\left(\tilde{a}_{33}+\tilde{a}_{55}\right) \\
& \tilde{a}_{3}=-\operatorname{det} \tilde{M}
\end{aligned}
$$

Then the equilibrium $E_{1}$ is hyperbolic sink, locally asymptotically stable, and a local attractor if 


$$
\tilde{a}_{1}>0, \quad \tilde{a}_{2}>0, \tilde{a}_{3}>0, \tilde{a}_{1} \tilde{a}_{2}>\tilde{a}_{3}
$$

Proof. Let $\lambda_{I}$ for $i=\{1,2,3,4,5\}$ denote the eigen-values corresponding to the Jacobianmatrix $J\left\{E_{1}\left[0,0,0,0, x_{5}\right]\right\}$ given by (4.3). Then the corresponding eigen-spectrum is given by the equation:

$$
\sigma\left(J\left\{E_{1}\left[0,0,0,0, \tilde{x}_{5}\right]\right\}\right)=\left\{\lambda_{i} \mid \lambda^{3}+\tilde{a}_{1} \lambda^{2}+\tilde{a}_{2} \lambda+\tilde{a}_{3}=0, i=1,2,3\right\} \cup\left\{\lambda_{4}, \lambda_{5}\right\}
$$

Where $\tilde{a}_{1}, \tilde{a}_{2}, \tilde{a}_{3}$ are specified in (5.9). But $\lambda_{4}=\tilde{a}_{11}=-k_{1}<0$ and $\lambda_{5}=\tilde{a}_{44}=-k_{4}<0$.

The conclusion of the theorem follows immediately after application of the Routh-Hurwicz criteria.

Clinical Implication 5.3. If the HAART protocol used for the AIDS patient is such that condition (5.9) is satisfied, then the patient experiences extreme immune system cytotoxicity in which the uninfected $\mathrm{CD}^{+}{ }^{+} \mathrm{T}$ cells, HIV-1 specific cytotoxic $\mathrm{CD}^{+} \mathrm{T}$ cells, HIV-1 virions, and HIV-1 infected $\mathrm{CD} 4^{+} \mathrm{T}$ cells are all decimated. This is not a therapeutically desirable clinical outcome, because the patient may become severely incapacitated. To avoid this scenario, the HAART therapy must implemented in such a way that criterion (5.9) is violated.

\subsection{Local Stability of $E_{3}=\left[\bar{x}_{1}, 0,0, \bar{x}_{4}, \bar{x}_{5}\right]$}

The local existence and local stability of the equilibrium $E_{3}=\left[\bar{x}_{1}, 0,0, \bar{x}_{4}, \bar{x}_{5}\right]$ will be analyzed in this subsection. The clinical relevance of the theoretical therapeutic criteria will be discussed.

Let $\sigma\left(J\left\{E_{3}\left[\bar{x}_{1}, 0,0, \bar{x}_{4}, \bar{x}_{5}\right]\right\}\right.$ be the eigen-spectrum for the Jacobian matrix $\left(J\left\{E_{3}\left[\bar{x}_{1}, 0,0, \bar{x}_{4}, \bar{x}_{5}\right]\right\}\right.$ such that $\sigma\left(J\left\{E_{3}\left[\bar{x}_{1}, 0,0, \bar{x}_{4}, \bar{x}_{5}\right]\right\}\right)=\left\{\lambda_{i}|\operatorname{det}| J\left(E_{3}\right)-\lambda I \mid=0, i=1,2,3,4,5\right\}$

\section{Theorem 5.4. Suppose}

(i) The local existence criteria $C_{3}$ holds for $E_{3}$

(ii) $\bar{x}_{1}=\frac{\beta_{1}}{\alpha_{2}}=\frac{1}{b_{4}}$

(iii) $\left\{\begin{array}{l}a_{1} \bar{x}_{1}\left(2-b_{1} \bar{x}_{1}\right) e^{-b_{1} \bar{x}_{1}}-k_{1}<0 \\ a_{2} \bar{x}_{1} e^{-b_{2} \bar{x}_{1}}-q_{2} \bar{x}_{1}-k_{2}-K_{1} \bar{x}_{4}-\frac{\xi_{2} \sigma_{2} \bar{x}_{5}}{\lambda_{2}+\bar{x}_{5}}<0\end{array}\right.$

(iv) $\bar{M}=\left[\begin{array}{ccc}\bar{a}_{33} & 0 & \bar{a}_{35} \\ \bar{a}_{43} & \bar{a}_{44} & 0 \\ \bar{a}_{53} & 0 & \bar{a}_{55}\end{array}\right]$

with

$$
\sigma\left(J\left(E_{3}\right)\right)=\left[\lambda_{i} \mid \lambda^{3}+\bar{a}_{1} \lambda^{2}+\bar{a}_{2} \lambda+\bar{a}_{3}=0, i=1,2,3\right] \cup\left[\lambda_{4}, \lambda_{5}\right]
$$


where

$$
\begin{aligned}
& \bar{a}_{1}=-\operatorname{Trace}(\bar{M}) \\
& \bar{a}_{2}=\operatorname{det}\left|\begin{array}{cc}
\bar{a}_{44} & 0 \\
0 & \bar{a}_{55}
\end{array}\right|+\operatorname{det}\left|\begin{array}{ll}
\bar{a}_{33} & \bar{a}_{35} \\
\bar{a}_{53} & \bar{a}_{55}
\end{array}\right|+\operatorname{det}\left|\begin{array}{cc}
\bar{a}_{33} & 0 \\
\bar{a}_{43} & \bar{a}_{44}
\end{array}\right| \\
& \bar{a}_{3}=-\operatorname{det} \bar{M} \\
& \lambda_{4}=\bar{a}_{11}<0 \\
& \lambda_{5}=\bar{a}_{22}<0
\end{aligned}
$$

Then the equilibrium $E_{3}$ exists locally and is a local attractor if

$$
\bar{a}_{1}>0, \bar{a}_{2}>0, \bar{a}_{3}>0, \bar{a}_{1} \bar{a}_{2}>\bar{a}_{3}
$$

Proof. Consider the Jacobian matrix (4.5) associated with the equilibrium $E_{3}$. By imposing the condition (ii) of the theorem and computing the eigen-values using the Laplace expansion technique, it can be observed that the eigen-values satisfy equation (5.10). The Routh-Hurwicz criteria when applied to the matrix $\bar{M}$ together with the conditions in (5.10) guarantees the local existence of $E_{3}$ as a local attractor.

\subsection{Persistence Analysis of HAART}

The pathophysiology of HIV-1 persistence during HAART is described elaborately in the paper by Pomerantz in [18]. In this subsection, the mathematical criteria for viral persistence during HAART will be derived. The interior rest point $E_{10}$ exists if and only if the system (4.1) exhibits persistence. This describes a situation in which the patient survives for a long time with AIDS. It is possible during HAART that, the HIV-1 virions cannot be annihilated but instead the system persists in a chronic configuration in which the blood plasma levels of the HIV-1 virions co-exist with the other clinically measurable parameters such as uninfected $\mathrm{CD}^{+} \mathrm{T}$ cells, the HIV-1 infected $\mathrm{CD}^{+} \mathrm{T}$ cells, HIV-1 specific $\mathrm{CD}^{+} \mathrm{T}$ cells, and HAART drug molecules. The criteria for existence of $E_{10}$ are listed in the following theorem.

Theorem 5.5. Let

(ii)

$$
\left\{\begin{array}{l}
m_{i}=\inf _{t \in\left[t_{0}, T\right]}\left\{a_{i} \breve{x}_{1} \breve{x}_{i} e^{-b_{i} \breve{x}_{1}}\right\} \text { where } i=\{1,2,4\} \\
L_{1}=\sup _{t \in\left[t_{0}, T\right]}\left\{\alpha_{1} \breve{x}_{3}+q_{1} \breve{x}_{2}+k_{1}\right\} \\
L_{2}=\sup _{t \in\left[t_{0}, T\right]}\left\{q_{2} \breve{x}_{1}+k_{2}+K_{1} \breve{x}_{4}+\frac{\xi_{2} \sigma_{2} \breve{x}_{2} \breve{x}_{5}}{\lambda_{2}+\breve{x}_{5}}\right\} \\
U_{1}=\sup _{t \in\left[t_{0}, T\right]}\left\{\beta_{1} \breve{x}_{3}\right\} \\
L_{3}=\sup _{t \in\left[t_{0}, T\right]}\left\{\alpha_{3} \breve{x}_{1}+\eta_{1} \breve{x}_{4}+k_{3}+\frac{\xi_{3} \sigma_{3}}{\lambda_{3}+\breve{x}_{5}}\right\} \\
m_{3}=\inf _{t \in\left[t_{0}, T\right]}\left\{\beta_{2} \breve{x}_{2} \breve{x}_{3}+\beta_{3} \breve{x}_{3}\right\} \\
L_{4}=\sup _{t \in\left[t_{0}, T\right]}\left\{K_{2} \breve{x}_{2}+\eta_{2} \breve{x}_{3}+k_{4}\right\} \\
L_{5}=\sup _{t \in\left[t_{0}, T\right]}\left\{\frac{\sigma_{0}}{\lambda_{0}+\breve{x}_{5}}+\frac{\sigma_{2} \breve{x}_{2}}{\lambda_{2}+\breve{x}_{5}}+\frac{\sigma_{3} \breve{x}_{3}}{\lambda_{3}+\breve{x}_{5}}+k_{5} \breve{x}_{5}\right\}
\end{array}\right.
$$




$$
\left\{\begin{array}{l}
\lim \inf x_{1} \geq \frac{S_{1}+m_{1}-e_{10}}{L_{1}}>0 \\
\liminf x_{2} \geq \frac{S_{2}+m_{2}-U_{1}-e_{20}}{L_{2}}>0 \\
\liminf x_{3} \geq \frac{S_{3}+m_{3}-e_{30}}{L_{3}}>0 \\
\liminf x_{4} \geq \frac{S_{4}+m_{4}-e_{40}}{L_{4}}>0 \\
\lim \inf x_{5} \geq \frac{D}{L_{5}}>0
\end{array}\right.
$$

Then the system $\left[x_{1}, x_{2}, x_{3}, x_{4}, x_{5}\right]$ will persist and the HAART therapeutic outcome $E_{10}=$ $\left[\breve{x}_{1}, \breve{x}_{2}, \breve{x}_{3}, \breve{x}_{4}, \breve{x}_{5}\right]$ exists.

Proof. For a proof refer to Nani and Jin $[7,15,16]$.

Clinical Implication 5.5. If uninfected $\mathrm{CD}^{+} \mathrm{T}$ cells, infected $\mathrm{CD} 4^{+} \mathrm{T}$ cells, the HIV-1 viral mRNA copies in the blood plasma, HIV-1 specific cytotoxic $\mathrm{CD}^{+} \mathrm{T}$ cells, and the HAART drug concentration are within certain thresholds, then the AIDS patient will live with AIDS. If any of the conditions listed in (5.11) are violated, the patient will experience unpredictable therapeutic outcomes including full-blown AIDS or spontaneous elimination of HIV-1 virions.

\section{Sufficient Criteria for Permanent Cure of AIDS during HAART}

In this section, the sufficient criteria for permanent annihilation of HIV-1 virions during HAART will be derived. The clinically desirable physiological outcomes are $E_{4}\left[x_{1}, 0,0,0, x_{5}\right]$ and $E_{3}=\left[x_{1}, 0\right.$, $0, x_{4}, x_{5}$. It must be recalled that $E_{4}$ corresponds to the physiological outcome in which HIV-1 infected $\mathrm{CD}^{+} \mathrm{T}$ cells, the HIV-1 virions in the blood plasma and HIV-1 specific CD8 ${ }^{+} \mathrm{T}$ cells are eliminated in the blood plasma of the AIDS patient. The sufficient criteria for $E_{4}$ will be discussed first.

\subsection{Global Stability of $E_{4}=\left[\hat{x}_{1}, 0,0,0, \hat{x}_{5}\right]$}

In this subsection, the global stability of $E_{4}=\left[\hat{x}_{1}, 0,0,0, \hat{x}_{5}\right]$ will be established in the space $\Omega=\left\{\left(x_{1}, x_{2}, x_{3}, x_{4}, x_{5}\right) \in \mathfrak{R}_{+}^{5} \mid 0 \leq x_{i} \leq \Phi_{i} i=1,2,3,4,5\right\} \quad$ with $\Phi_{i}$ defined in (3.2).

Let

$$
S_{+}^{x_{1} x_{5}}=\left\{\left(x_{1}, x_{5}\right) \in \mathfrak{R}_{+}^{2} \mid 0 \leq x_{i} \leq \Phi_{i} \quad i=1,5\right\} \subset \Omega
$$

The global asymptotic stability of $E_{4}$ in $\Omega$ will be established respect to the flow emanating from int $S_{+}^{x_{1} x_{5}} \subset$ int $R_{+}^{x_{1} x_{5}}$. 
The model equations (4.1) correspondingly reduce to the following:

$$
\left\{\begin{array}{l}
\dot{x}_{1}=S_{1}+a_{1} x_{1}^{2} e^{-b_{1} x_{1}}-k_{1} x_{1}-e_{10} \\
\dot{x}_{5}=D-\frac{\sigma_{0} x_{5}}{\lambda_{0}+x_{5}}-k_{5} x_{5} \\
x_{i}\left(t_{0}\right)=x_{i 0} \text { for } \quad i=\{1,5\}
\end{array}\right.
$$

Let $N$ be a neighborhood of a point in int $S_{+}^{x_{1} x_{5}} \subset \Omega$. Choose a functional $\mathrm{V}$ on $N$ such that

(i) $\quad V$ is positive definite with respect to $E_{4}$

(ii) $\quad V \rightarrow \infty$ as $\left\|x_{i}\right\| \rightarrow \partial R_{+}^{x_{1} x_{5}} i=\{1,5\}$

(iii) $\quad V \in C^{1}\left(R^{2}, R\right)$ and bounded below

(iv) $V$ is a Liapunov functional for (6.1)

(v) $\quad V\left(E_{4}\right)=0$

(vi) $\exists \eta>0$ such that $V\left(x_{i}\right)>0$ wherever $x_{i} \in B_{\eta}\left(E_{4}\right)$ for $\hat{x} \neq x$ where $B_{\eta}\left(x_{i}\right)=\left\{x \in R_{+}^{x_{1} x_{5}}\left\|x-\bar{x}_{i}\right\|<\eta\right\}$

(v) $\quad \stackrel{*}{V} \leq 0$ along the solution trajectories of (6.1)

Consider the Liapunov functional:

$$
\begin{aligned}
V & :=\sum \frac{1}{2} \hat{c}_{i}\left(x_{i}-\hat{x}_{i}\right)^{2} \\
& \text { where } i=\{1,5\} \text { and } \hat{c}_{i} \in R_{+}=(0, \infty)
\end{aligned}
$$

The derivative of $\mathrm{V}$ along the solution trajectories of the model equations yields the result:

$$
\begin{aligned}
& \stackrel{*}{V}=\hat{c}_{1}\left(x_{1}-\hat{x}_{1}\right) \dot{x}_{1}+\hat{c}_{5}\left(x_{5}-\hat{x}_{5}\right) \dot{x}_{5} \\
& =\hat{c}_{1}\left(x_{1}-\hat{x}_{1}\right)\left(S_{1}+a_{1} x_{1}^{2} e^{-b_{1} x_{1}}-k_{1} x_{1}-e_{10}\right)+ \\
& \hat{c}_{5}\left(x_{5}-\hat{x}_{5}\right)\left(D-\frac{\sigma_{0} x_{5}}{\lambda_{0}+x_{5}}-k_{5} x_{5}\right)
\end{aligned}
$$

But at a steady state, the following equations hold.

$$
\left\{\begin{array}{l}
S_{1}-e_{10}=k_{1} \hat{x}_{1}-a_{1} \hat{x}_{1}^{2} e^{-b_{1} \hat{x}_{1}} \\
D=\frac{\sigma_{0} \hat{x}_{5}}{\lambda_{0}+\hat{x}_{5}}-k_{5} x_{5}
\end{array}\right.
$$

Define the following Lebesgue measurable, functions which are of bounded variation: 


$$
\begin{aligned}
& G\left(x_{1}\right)=a_{1} x_{1}^{2} e^{-b_{1} x_{1}} \\
& L\left(x_{5}\right)=\frac{\sigma_{0} x_{5}}{\lambda_{0}+x_{5}}
\end{aligned}
$$

$$
\begin{aligned}
V^{*}= & \hat{c}_{1}\left(x_{1}-\hat{x}_{1}\right) \dot{x}_{1}+\hat{c}_{5}\left(x_{5}-\hat{x}_{5}\right) \dot{x}_{5} \\
= & \hat{c}_{1}\left(x_{1}-\hat{x}_{1}\right)\left(-a_{1} \hat{x}_{1}^{2} e^{-b_{1} x_{1}}+k_{1} \hat{x}_{1}+a_{1} x_{1}^{2} e^{-b_{1} x_{1}}-k_{1} x_{1}\right)+\hat{c}_{5}\left(x_{5}-\hat{x}_{5}\right)\left(\frac{\sigma_{0} \hat{x}_{5}}{\lambda_{0}+\hat{x}_{5}}-\frac{\sigma_{0} x_{5}}{\lambda_{0}+x_{5}}\right) \\
& \quad+\hat{c}_{5} k_{5}\left(x_{5}-\hat{x}_{5}\right)\left(\hat{x}_{5}-x_{5}\right) \\
= & \left.\hat{c}_{1}\left(x_{1}-\hat{x}_{1}\right)\left[G\left(x_{1}\right)-G\left(\hat{x}_{1}\right)\right]+\hat{c}_{1}\left(x_{1}-\hat{x}_{1}\right)\left(k_{1} \hat{x}_{1}-k_{1} x_{1}\right)\right] \\
& \quad+\hat{c}_{5}\left(x_{5}-\hat{x}_{5}\right)\left[L\left(\hat{x}_{5}\right)-L\left(x_{5}\right)\right]-\hat{c}_{5} k_{5}\left(x_{5}-\hat{x}_{5}\right)^{2}
\end{aligned}
$$

$\stackrel{*}{V}=-\hat{c}_{1} k_{1}\left(x_{1}-\hat{x}_{1}\right)^{2}+\hat{c}_{1}\left(x_{1}-\hat{x}_{1}\right)\left[G\left(x_{1}\right)-G\left(\hat{x}_{1}\right)\right]-\hat{c}_{5}\left(x_{5}-\hat{x}_{5}\right)\left[L\left(x_{5}\right)-L\left(\hat{x}_{5}\right)\right]-\hat{c}_{5} k_{5}\left(x_{5}-\hat{x}_{5}\right)^{2}$

Let

$$
\begin{aligned}
& v_{1}=x_{1}-\hat{x}_{1} \\
& v_{2}=x_{5}-\hat{x}_{5}
\end{aligned}
$$

and

$$
X=\left[\begin{array}{l}
v_{1} \\
v_{2}
\end{array}\right] \in R_{+}^{2}
$$

and define $M=\left\{m_{i j}\right\} \in M_{2 \times 2}(R)$ such that

then

$$
M=\left[\begin{array}{cc}
m_{11} & \frac{1}{2} m_{12} \\
\frac{1}{2} m_{21} & m_{22}
\end{array}\right]
$$

$$
\begin{aligned}
\stackrel{*}{V} & :=m_{11} v_{1}^{2}+\frac{1}{2} m_{12} v_{1} v_{2}+\frac{1}{2} m_{21} v_{2} v_{1}+m_{22} v_{2}^{2} \\
& =X^{T} M X
\end{aligned}
$$

Where $X^{T}$ denotes the transpose of $X$ and ${ }^{*}$ is negative definite if satisfy the conditions of $m_{11}<0$ and $\operatorname{det}(M)>0$.

In particular, the $\left[m_{i j}\right]_{2 \times 2}$ are defined as follows: 


$$
\left\{\begin{array}{l}
m_{11}:=-\left[\hat{c}_{1} k_{1}-\hat{c}_{1}\left(\frac{G\left(x_{1}\right)-G\left(\hat{x}_{1}\right)}{x_{1}-\hat{x}_{1}}\right)\right] \\
m_{12}=m_{21}=0 \\
m_{22}:=-\hat{c}_{5}\left[\frac{L\left(x_{5}\right)-L\left(\hat{x}_{5}\right)}{x_{5}-\hat{x}_{5}}\right]-\hat{c}_{5} k_{5}
\end{array}\right.
$$

As the flow dynamics approaches the steady state $E_{4}=\left[\hat{x}_{1}, 0,0,0, \hat{x}_{5}\right]$, the following conditions hold:

$$
\begin{aligned}
& \lim _{x_{1} \rightarrow \hat{x}_{1}} m_{11}=-\left[\hat{c}_{1}\left(k_{1}-G_{1}^{\prime}\left(\hat{x}_{1}\right)\right)\right] \\
& \lim _{x_{2} \rightarrow \hat{x}_{2}} m_{22}=-\hat{c}_{5}\left(L^{\prime}\left(\hat{x}_{5}\right)-k_{5}\right)
\end{aligned}
$$

where

$$
\begin{aligned}
& G^{\prime}\left(\hat{x}_{1}\right)=a_{1} \hat{x}_{1} e^{-b_{1} \hat{x}_{1}}\left(2-b_{1} \hat{x}_{1}\right) \\
& L^{\prime}\left(\hat{x}_{5}\right)=\frac{\sigma_{0} \lambda_{0}}{\left(\lambda_{0}+\hat{x}_{5}\right)^{2}}>0
\end{aligned}
$$

In particular,

$$
G^{\prime}\left(\hat{x}_{1}\right)\left\{\begin{array}{lll}
>0 & \text { if } & \hat{x}_{1}<2 / b_{1} \\
= & 0 \text { if } & \hat{x}_{1}=2 / b_{1} \\
<0 & \text { if } & \hat{x}_{1}>2 / b_{1}
\end{array}\right.
$$

The sufficient criteria for the global asymptotic stability of $E_{4}$ are specified in the following theorem.

\section{Theorem 6.1 Let}

(i) Conditions of Theorem 5.1 holds

(ii) $\hat{x}_{1} \leq \frac{2}{b_{1}}$ and $k_{1}>G^{\prime}\left(\hat{x}_{1}\right)$

Then the equilibrium $E_{4}$ is a globally asymptotically stable with respect to the flow emanating from int $S_{+}^{x_{1} x_{5}} \subset \Omega$.

Proof. The condition (i) of the theorem guarantees the local existence of $E_{4}$ and establishes that $E_{4}$ is a local attractor. The global asymptotic stability of $E_{4}$ can be shown by invoking the Gantmacher criteria [30] for negative definiteness of matrix $M$ as defined in (6.10). In particular, $V^{*}$ is negative definite if $m_{11}<0$ and $\operatorname{det} M>0$. But $m_{11}=-\hat{c}_{1}\left[k_{1}-G_{1}^{\prime}\left(\hat{x}_{1}\right)\right]$ is less than zero if condition (ii) of the theorem hypothesis holds. However, $\operatorname{det} M>0$ since the product $m_{11} m_{22}>0$. Thus, $V<0$ and hence $E_{4}$ is a global attractor. This completes the proof. 


\section{Comments on Theorem 6.1}

(i) If $G\left(\hat{x}_{1}\right)>0$ then $k_{1}>G\left(\hat{x}_{1}\right)$ and $\hat{x}_{1}>\frac{2}{b_{1}}$ constitute sufficient condition for global asymptotic stability of $E_{4}$.

(ii) If $G\left(\hat{x}_{1}\right)<0$, then $\hat{x}_{1}>\frac{2}{b_{1}}$ is a sufficient condition for global asymptotic stability of $E_{4}$.

(iii) If $G\left(\hat{x}_{1}\right)=0$ then $\hat{x}_{1}=\frac{2}{b_{1}}$ is a sufficient condition for global asymptotic stability of $E_{4}$.

\section{Clinical Implication 6.1}

(i) The function $G\left(\hat{x}_{1}\right)$ is a measure of the rate at which the $\mathrm{CD} 4^{+} \mathrm{T}$ cells $\left(x_{1}\right)$ are activated by interleukin-2 (IL-2) in an autocrine process when the physiological flow during HAART tends to $E_{4}$. It is possible to attach a biochemical measure to coefficient $a_{1}$ and $b_{1}$ of $G\left(\hat{x}_{1}\right)$. This can be done as follows:

$$
G\left(\hat{x}_{1}\right)=a_{1} \hat{x}_{1}^{2} e^{-b_{1} \hat{x}_{1}}=\frac{a_{1} \hat{x}_{1}^{2}}{e^{b_{1} \hat{x}_{1}}}=\frac{a_{1} \hat{x}_{1} \hat{x}_{1}}{1+b_{1} \hat{x}_{1}+\frac{\left(b_{1} \hat{x}_{1}\right)^{2}}{2 !}+\ldots} \approx \frac{a_{1} \hat{x}_{1} \hat{x}_{1}}{1+b_{1} \hat{x}_{1}}=\frac{\frac{a_{1}}{b_{1}} \hat{x}_{1} \hat{x}_{1}}{\frac{1}{b_{1}}+\hat{x}_{1}}=\frac{V_{\max } \hat{x}_{1} \hat{x}_{1}}{K_{m}+\hat{x}_{1}}
$$

where $K_{m}=\frac{1}{b_{1}}$ is approximately equal to the Michaelis-Menten constant of the $\mathrm{CD} 4^{+} \mathrm{T}$ cells activation reaction by interleukin-2, and $V_{\max }=\frac{a_{1}}{b_{1}}$ represents the maximal velocity of the $\mathrm{CD} 4^{+} \mathrm{T}$ cells IL-2 activation reaction.

(ii) Note that $k_{1}$ is the rate of degradation and decay of the $\mathrm{CD} 4^{+} \mathrm{T}$ cells during AIDS. Thus the theorem implies that if the rate of degradation of $\mathrm{CD}^{+} \mathrm{T}$ cells is greater than the rate of IL-2 activation of $\mathrm{T}$ cells and if in addition, the number of the CD4+ T cells are such that $\hat{x}_{1}<2 K_{m}$, then the patient will be cured of AIDS.

\subsection{Global Stability Analysis for $E_{3}=\left[\bar{x}_{1}, 0,0, \bar{x}_{4}, \bar{x}_{5}\right]$}

In this subsection, the other clinical desirable equilibrium $E_{3}=\left[\bar{x}_{1}, 0,0, \bar{x}_{4}, \bar{x}_{5}\right]$ is analyzed. The aim is to find the sufficient criteria under which the HIV-1 virions in the blood plasma, the HIV-1 infected $\mathrm{CD} 4^{+} \mathrm{T}$ cells are annihilated but the HIV-1 specific $\mathrm{CD} 8^{+} \mathrm{T}$ cells will persist as memory $\mathrm{T}$ cells as well as the $\mathrm{CD} 4^{+} \mathrm{T}$ cells and some drug residues will remain in the AIDS patient. It is expected that the $\mathrm{CD} 4^{+} \mathrm{T}$ cells will eventually repopulate to their carrying capacity whereas the drug residues will ultimately dissipate. The analysis will be similar to the preceding one for $E_{4}$. 
The restriction of the model equations (4.1) to the space $S_{+}^{x_{1} x_{4} x_{5}}=\left\{\left(x_{1}, x_{4}, x_{5}\right) \in \Re_{+}^{3} \mid 0 \leq x_{i} \leq \Phi_{i} \quad i=1,4,5\right\} \subset \Omega$ leads to the following equations.

$$
\left\{\begin{array}{l}
\dot{x}_{1}=S_{1}+a_{1} x_{1}^{2} e^{-b_{1} x_{1}}-k_{1} x_{1}-e_{10} \\
\dot{x}_{4}=S_{4}+a_{4} x_{1} x_{4} e^{-b_{4} x_{1}}-k_{4} x_{4}-e_{40} \\
\dot{x}_{5}=D-\frac{\sigma_{0} x_{5}}{\lambda_{0}+x_{5}}-k_{5} x_{5} \\
x_{i}\left(t_{0}\right)=x_{i 0} \text { for } \quad i=\{1,4,5\}
\end{array}\right.
$$

The global stability of $E_{3}=\left[\bar{x}_{1}, 0,0, \bar{x}_{4}, \bar{x}_{5}\right]$ will be established with respect to the flow emanating from int $S_{+}{ }^{x_{1} x_{4} x_{5}} \subset \Omega$.

Consider the Liapnnov functional:

$$
\begin{aligned}
V & :=\sum \frac{1}{2} \bar{c}_{i}\left(x_{i}-\overline{x_{i}}\right)^{2} \\
& \text { where } i=\{1,4,5\} \text { and } \overline{c_{i}} \in R_{+}=(0, \infty)
\end{aligned}
$$

The derivative of $\mathrm{V}$ along the solution curves of the model equations yields the result:

$$
\stackrel{*}{V}=\bar{c}_{1}\left(x_{1}-\bar{x}_{1}\right) \dot{x}_{1}+\bar{c}_{4}\left(x_{4}-\bar{x}_{4}\right) \dot{x}_{4}+\bar{c}_{5}\left(x_{5}-\bar{x}_{5}\right) \dot{x}_{5}
$$

Thus a steady state, $\dot{x}_{i}=0$ and the following equations hold.

Thus

$$
\begin{aligned}
& \left\{\begin{array}{l}
S_{1}-e_{10}=k_{1} \bar{x}_{1}-a_{1} \bar{x}_{1}^{2} e^{-b_{1} \bar{x}_{1}} \\
S_{4}-e_{40}=k_{4} \bar{x}_{4}-a_{4} \bar{x}_{1} \bar{x}_{4} e^{-b_{4} \bar{x}_{1}} \\
D=\frac{\sigma_{0} \bar{x}_{5}}{\lambda_{0}+\bar{x}_{5}}+k_{5} \bar{x}_{5}
\end{array}\right. \\
& \stackrel{*}{V}=\bar{c}_{1} k_{1}\left(x_{1}-\bar{x}_{1}\right)\left(\bar{x}_{1}-x_{1}\right) \\
& +\bar{c}_{1}\left(x_{1}-\bar{x}_{1}\right)\left[G\left(\bar{x}_{1}\right)-G\left(x_{1}\right)\right] \\
& +\bar{c}_{4} k_{4}\left(x_{4}-\bar{x}_{4}\right)\left(\bar{x}_{4}-x_{4}\right) \\
& +\bar{c}_{4}\left(x_{4}-\bar{x}_{4}\right)\left[F\left(\bar{x}_{1}, \bar{x}_{4}\right)-F\left(x_{1}, x_{4}\right)\right] \\
& +\bar{c}_{5} \sigma_{0}\left[L\left(\bar{x}_{5}\right)-L\left(x_{5}\right)\right] \\
& \text { where } \\
& G\left(x_{1}\right)=a_{1} x_{1}^{2} e^{-b_{1} x_{1}} \\
& F\left(x_{1}, x_{4}\right)=a_{4} x_{1} x_{4} e^{-b_{4} x_{1}} \\
& L\left(x_{5}\right)=\frac{\sigma_{0} x_{5}}{\lambda_{0}+x_{5}}
\end{aligned}
$$


The functions $G, F, L$ are continuous, Lebesgue measurable, and of bounded variations.

$$
\text { Let } \quad \stackrel{*}{V}=X^{T} C X
$$

where

$$
X=\left[\begin{array}{l}
u_{1} \\
u_{2} \\
u_{3}
\end{array}\right] \in R_{+}^{3}
$$

and

$$
\begin{aligned}
& u_{1}=x_{1}-\bar{x}_{1} \\
& u_{2}=x_{4}-\bar{x}_{4} \\
& u_{3}=x_{5}-\bar{x}_{5}
\end{aligned}
$$

such that $X^{T}$ denotes the transpose of $X$. Define $C \in M_{3 \times 3}(R)$ such that

$$
C=\left[\begin{array}{ccc}
c_{11} & \frac{1}{2} c_{12} & \frac{1}{2} c_{13} \\
\frac{1}{2} c_{12} & c_{22} & \frac{1}{2} c_{23} \\
\frac{1}{2} c_{13} & \frac{1}{2} c_{23} & c_{33}
\end{array}\right]
$$

Now

$$
\begin{aligned}
V^{*} & :=c_{11} u_{1}^{2}+\frac{1}{2} c_{12} u_{1} u_{2}+\frac{1}{2} c_{13} u_{1} u_{3} \\
& +\frac{1}{2} c_{12} u_{2} u_{1}+c_{22} u_{2}^{2}+\frac{1}{2} c_{23} u_{2} u_{3} \\
& +\frac{1}{2} c_{13} u_{3} u_{1}+\frac{1}{2} c_{23} u_{3} u_{2}+c_{33} u_{3}^{2}
\end{aligned}
$$

Where the $\left[c_{i j}\right]_{3 \times 3}$ are defined as follows:

$$
\left\{\begin{array}{l}
c_{11}:=-\left[\bar{c}_{1} k_{1}-\bar{c}_{1}\left(\frac{G\left(x_{1}\right)-G\left(\bar{x}_{1}\right)}{x_{1}-\bar{x}_{1}}\right)\right] \\
c_{12}:=-\bar{c}_{4}\left[\frac{F\left(x_{1}, x_{4}\right)-F\left(\bar{x}_{1}, \bar{x}_{4}\right)}{x_{1}-\bar{x}_{1}}\right]=c_{21} \\
c_{13}=c_{31}=0 \\
c_{22}=-\bar{c}_{4} k_{4} \\
c_{23}=c_{32}=0 \\
c_{33}:=-\bar{c}_{5}\left[\frac{L\left(x_{5}\right)-L\left(\bar{x}_{5}\right)}{x_{5}-\bar{x}_{5}}\right]-\bar{c}_{5} k_{5}
\end{array}\right.
$$

As the flow associated with the model equations approaches $E_{3}=\left[\bar{x}_{1}, 0,0, \bar{x}_{4}, \bar{x}_{5}\right]$, the matrix entries $\left[c_{i j}\right]_{3 \times 3}$ have the following form: 


$$
\begin{aligned}
& \lim _{x_{1} \rightarrow \bar{x}_{1}} c_{11}=-\left[\bar{c}_{1} k_{1}-\bar{c}_{1} G^{\prime}\left(\bar{x}_{1}\right)\right] \\
& \lim _{x_{1} \rightarrow \bar{x}_{1}} c_{12}=-\bar{c}_{4}\left[F_{x_{1}}\left(\bar{x}_{1}, \bar{x}_{4}\right)\right] \\
& \lim _{x_{4} \rightarrow \bar{x}_{4}} c_{22}=-\bar{c}_{4} k_{4} \\
& \lim _{x_{5} \rightarrow \bar{x}_{5}} c_{33}=-\bar{c}_{5} L^{\prime}\left(\bar{x}_{5}\right)-\bar{c}_{5} k_{5}
\end{aligned}
$$

But it can be shown that

$$
\begin{aligned}
& F_{x_{1}}\left(\bar{x}_{1}, \bar{x}_{4}\right)=a_{4} \bar{x}_{4} e^{-b_{4} \bar{x}_{1}}\left(1-\bar{x}_{1} b_{4}\right) \\
& G^{\prime}\left(\bar{x}_{1}\right)=a_{1} \bar{x}_{1} e^{-b_{1} \bar{x}_{1}}\left(2-\bar{x}_{1} b_{1}\right) \\
& L^{\prime}\left(\bar{x}_{5}\right)=\frac{\sigma_{0} \lambda_{0}}{\left(\lambda_{0}+\bar{x}_{5}\right)^{2}}>0
\end{aligned}
$$

In particular, (cf. Nani and Jin $[7,15,16])$

$$
F_{x_{1}}\left(\bar{x}_{1}, \bar{x}_{4}\right)\left\{\begin{array}{l}
>0 \text { if } \bar{x}_{1}<\frac{1}{b_{4}} \\
=0 \text { if } \bar{x}_{1}=\frac{1}{b_{4}} \\
<0 \text { if } \bar{x}_{1}>\frac{1}{b_{4}}
\end{array}\right.
$$

Similarly,

$$
G^{\prime}\left(\bar{x}_{1}\right)\left\{\begin{array}{l}
>0 \text { if } \bar{x}_{1}<\frac{2}{b_{1}} \\
=0 \text { if } \bar{x}_{1}=\frac{2}{b_{1}} \\
<0 \text { if } \bar{x}_{1}>\frac{2}{b_{1}}
\end{array}\right.
$$

Using the specifications in (4.31), the matrix $C$ has the form

$$
C=\left[\begin{array}{ccc}
c_{11} & \frac{1}{2} c_{12} & 0 \\
\frac{1}{2} c_{12} & c_{22} & 0 \\
0 & 0 & c_{33}
\end{array}\right]
$$

The matrix $C$ is negative definite if the following criteria hold:

(i) $\operatorname{det} c_{11}<0$ or $c_{11}<0$

(ii) $\operatorname{det}\left|\begin{array}{cc}c_{11} & \frac{1}{2} c_{12} \\ \frac{1}{2} c_{12} & c_{22}\end{array}\right|>0$ or $\quad c_{11} c_{22}-\frac{1}{4}\left(c_{12}\right)^{2}>0$

(iii) $\operatorname{det}\left|\begin{array}{ccc}c_{11} & \frac{1}{2} c_{12} & 0 \\ \frac{1}{2} c_{12} & c_{22} & 0 \\ 0 & 0 & c_{33}\end{array}\right|<0 \quad$ or $\quad c_{33}\left[c_{11} c_{22}-\frac{1}{4}\left(c_{12}\right)^{2}\right]<0$ 
Theorem 6.2 Let $\left\{x_{1}, x_{4}, x_{5}\right\} \in S_{+}{ }^{x_{1} x_{4} x_{5}} \subset \Omega$. Consider the patho-physiological equilibrium $E_{3}=\left[\bar{x}_{1}, 0,0, \bar{x}_{4}, \bar{x}_{5}\right]$ such that

$$
\begin{aligned}
& \text { (i) } \bar{x}_{1}=\frac{1}{b_{4}}<\frac{2}{b_{1}} \\
& \text { (ii) } k_{1}>G^{\prime}\left(\bar{x}_{1}\right)>0
\end{aligned}
$$

Then $E_{3}=\left[\bar{x}_{1}, 0,0, \bar{x}_{4}, \bar{x}_{5}\right]$ is globally asymptotically stable with respect to the flow emanating from $S_{+}{ }^{x_{1} x_{4} x_{5}} \subset \Omega$. In particular $E_{3}$ is a global attractor. Consequently, all the HIV-1 virions will be permanently annihilated in the AIDS patient.

Proof. The patho-physiological equilibrium $E_{3}$ is a global attractor if ${ }^{*}=X^{T} C X$ is negative definite, or equivalently if the matrix $C$ is negative definite. But $C$ is negative definite if conditions (6.27) (i), (ii), (iii) hold (cf. Gantmacher [30]). Note that (6.27) (i) holds if $k_{1}>G^{\prime}\left(\bar{x}_{1}\right)$ and $\bar{x}_{1}<\frac{2}{b_{1}}$. If $\bar{x}_{1}=\frac{1}{b_{4}}$, then $c_{12}=0$, which leads to the fulfillment of (6.27) (ii). But $c_{33}<0$ and $c_{12}$ $=0$ whereas $c_{11} c_{22}>0$. Thus condition (6.27) (iii) is satisfied. As a result, $\stackrel{*}{V}$ is negative definite. Therefore, $E_{3}$ becomes a global attractor and hence the theorem follows.

\section{Clinical Implications 6.2}

(i) Theorem 6.2 gives the sufficient theoretical criteria for the HIV-1 infected $\mathrm{CD} 4{ }^{+} \mathrm{T}$ cells and HIV-1 virions in the blood plasma to be eradicated during HAART. This theorem also provides the conditions under which HIV-1 specific $\mathrm{CD} 8^{+} \mathrm{T}$ cells will persist as memory cells after the HAART therapy.

(ii) It is possible to express the conditions of Theorem 6.2 in terms of clinically measurable biophysical parameters. By using Taylor expansions of $e^{-b_{4} \bar{x}_{1}}$ $\bar{x}_{1}=\frac{1}{b_{4}}<\frac{2}{b_{1}}$ is approximately equivalent to the expression and $e^{-b_{1} \bar{x}_{1}}$, the condition

$$
\bar{x}_{1}=K_{m}^{C D 8^{+}}<2 K_{m}^{C D 4^{+}}
$$

This can be interpreted to mean that HAART will cure the AIDS patient if the number density of uninfected $\mathrm{CD}^{+} \mathrm{T}$ cells is equal to the Michaelis-Menten constant of the IL-2 activation of the $\mathrm{CD}^{+} \mathrm{T}$ cells, which in turn has to be less than twice Michaelis-Menten constant of the IL-2 activation of the $\mathrm{CD} 4^{+} \mathrm{T}$ cells.

Corollary 6.2 Suppose $\bar{x}_{1}=\frac{1}{b_{4}}=\frac{2}{b_{1}}$

Then the physiological steady state $E_{3}=\left[\bar{x}_{1}, 0,0, \bar{x}_{4}, \bar{x}_{5}\right]$ is a global attractor.

Proof. The conditions (6.27)(i),(ii), (iii) hold if the theorem hypothesis is satisfied. This makes the matrix $C$ in (6.19) negative definite. Consequently, the equilibrium becomes global attractor. 
It is possible to combine Theorem 6.2 and Corollary 6.2 to obtain the combined criteria:

$$
\begin{aligned}
\bar{x}_{1}=\frac{1}{b_{4}} \leq \frac{2}{b_{1}} & \\
& \bar{x}_{1} \approx K_{m}^{C D 8+} \leq 2 K_{m}^{C D 4+}
\end{aligned}
$$

\section{Simulations and Discussion of Results}

Investigative computer simulations will be presented and discussed. These simulations are performed using clinically plausible hypothetical patient patho-physiological parametric configurations. In particular, the simulations executed for the non-autonomous version of the model equations as displayed in (7.1).

$$
\left\{\begin{array}{l}
\dot{x}_{1}=S_{1}+a_{1} x_{1}^{2} e^{-b_{1} x_{1}}-\alpha_{1} x_{1} x_{3}-q_{1} x_{1} x_{2}-k_{1} x_{1}-e_{10} \\
\dot{x}_{2}=S_{2}+a_{2} x_{1} x_{2} e^{-b_{2} x_{1}}+\alpha_{2} x_{1} x_{3}-q_{2} x_{1} x_{2}-k_{2} x_{2}-\beta_{1} x_{3}-K_{1} x_{2} x_{4}-e_{20}-\frac{\xi_{2} \sigma_{2} x_{2} x_{5}}{\lambda_{2}+x_{5}} \\
\dot{x}_{3}=S_{3}+\beta_{2} x_{2} x_{3}+\beta_{3} x_{3}-\alpha_{3} x_{1} x_{3}-\eta_{1} x_{3} x_{4}-k_{3} x_{3}-e_{30}-\frac{\xi_{3} \sigma_{3} x_{3} x_{5}}{\lambda_{3}+x_{5}} \\
\dot{x}_{4}=S_{4}+a_{4} x_{1} x_{4} e^{-b_{4} x_{1}}-K_{2} x_{2} x_{4}-\eta_{2} x_{3} x_{4}-k_{4} x_{4}-e_{40} \\
\dot{x}_{5}=D f(t)-\frac{\sigma_{0} x_{5}}{\lambda_{0}+x_{5}}-\frac{\sigma_{2} x_{2} x_{5}}{\lambda_{2}+x_{5}}-\frac{\sigma_{3} x_{3} x_{5}}{\lambda_{3}+x_{5}}-k_{5} x_{5} \\
f(t)=|\lceil\sin 5 t\rceil| \\
x_{i}\left(t_{0}\right)=x_{i 0} \quad \text { for } \quad i=\{1,2,3,4,5\}
\end{array}\right.
$$

The drug input function $f(t)$ is a technical construct such as to generate the rectangle pulse function as shown in Section 3.1. It represents a periodically calibrated transdermal or intravenous or caplet drug matrix input with period $\omega$. In particular,

$$
f(t)=f(t+\omega) \quad \text { where } \omega=6.25 \text { days }
$$

It should be noted that the system (4.1) represents HAART during constant continuous caplet drug matrix input which is different from the scenarios used in the simulations of this section.

The complete mathematical analysis of the non-autonomous system (7.1) using Floquet Theory and Theory of non-linear periodic Dynamical Systems will be done in a future publication.

The numerical estimates used are variations of estimates published in the references: $[3,4,11,19,20,23]$. Some techniques for estimating HIV-I dynamical parameters includeMonte Carlo simulation and polynomial regression analysis. The investigative computer simulations elucidate several dynamical aspects of HIV-1 AIDS dynamics which are based on the respective parametric configurations. The $\mathrm{x}$-axis of the simulation graphs is calibrated in months.

\subsection{Simulation Results for Hypothetical AIDS patient \#1}

The AIDS patho-physiological parametric configuration of hypothetical patient \#1 is denoted by $P_{1}$ and exhibited in Table 1. The simulation results show that the HAART protocol impairs HIV-1 
dynamics in this patient leading to eradication of plasma HIV-1 viremia. The simulation results for patient \#1 are exhibited in Fig. 1. It can observed that the HIV-1 infected CD ${ }^{+} \mathrm{T}$ cells are eradicated in this patient with the use of anti-AIDS pharmaco-therapeutic drug protocols. In addition, patient \#1 experiences HAART- induced immune system reconstitution as the uninfected $\mathrm{CD}^{+} \mathrm{T}$ cells and HIV-1 specific $\mathrm{CD} 8^{+} \mathrm{T}$ cells repopulate.

Table 1. Hypothetical AIDS patient parametric configuration $\boldsymbol{P}_{1}$

\begin{tabular}{|c|c|c|c|c|}
\hline $\begin{array}{l}S_{1}=800 / \mathrm{day} / \mu l \\
a_{1}=0.15 / \mathrm{day} / \mathrm{cell} / \mu l \\
b_{1}=0.019 / \mathrm{cell} / \mu l \\
\alpha_{1}= \\
0.5 / \text { day } / \mathrm{virion} / \mu l \\
k_{1}=0.0005 / \mathrm{day} / \mu l \\
q_{1}= \\
0.00045 / \mathrm{day} / \mu \mathrm{l} / \mathrm{cell} \\
\mathrm{e}_{10}= \\
0.0025 \mathrm{cells} / \mathrm{day} / \mu l \\
x_{10}=500 \mathrm{cells} / \mu l\end{array}$ & $\begin{array}{l}S_{2}=800 / \mathrm{day} / \mu l \\
a_{2}=0.03 / \mathrm{day} / \mathrm{cell} / \mu l \\
b_{2}=0.004 / \mathrm{cell} / \mu l \\
\alpha_{2}=0.5 / \mathrm{day} / \mathrm{virion} / \mu l \\
k_{2}=0.005 / \mathrm{day} / \mu l \\
q_{2}= \\
0.00001 / \mathrm{day} / \mu l / \mathrm{cell} \\
\beta_{1}=1.05 \\
\text { virons } / \mathrm{CD} 4^{+} / \mathrm{day} \\
K_{1}=0.0001 / \mathrm{day} / \mu l \\
e_{20}=0.005 \\
\text { cells } / \text { day } / \mu l \\
\xi_{2}=0.85 \\
x_{20}=400 \mathrm{cells} / \mu l\end{array}$ & $\begin{array}{l}S_{3}=10 / \mathrm{day} / \mu l \\
\beta_{2}=0.0015 \\
\text { virons } / \mathrm{CD}^{+} / \mathrm{day} / \\
\mu l \\
\beta_{3}=1.05 \\
\text { virons } / \mathrm{CD} 4^{+} / \text {day } \\
\alpha_{3}=0.027 \\
/ \text { day } / \text { virion } / \mu l \\
k_{3}= \\
0.0001 / \mathrm{day} / \mu l \\
e_{30}=0.0001 / \mathrm{day} \\
\eta_{1}=0.25 \\
\xi_{3}=0.001 \\
x_{30}=500 \text { cells } / \mu l\end{array}$ & $\begin{array}{l}S_{4}=10 / \mathrm{day} / \mu l \\
a_{4}=0.35 \\
/ \text { day } / \mathrm{cell} / \mu l \\
b_{4}=0.01 / \mathrm{cell} / \mu l \\
K_{2}=0.0024 \\
/ \text { day } / \mu l \\
k_{4}=0.08 / \mathrm{day} / \mu l \\
e_{40}=0.0002 \\
\text { cells } / \text { day } / \mu l \\
\eta_{2}=0.45 \\
x_{40}=730 \text { cells } / \mu l\end{array}$ & $\begin{array}{l}\mathrm{D}=5000 \text { units } \\
\sigma_{0}=0.5 \mathrm{mg} / \text { day } \\
\sigma_{2}=30 \mathrm{mg} / \text { day } \\
\sigma_{3}=5 \mathrm{mg} / \text { day } \\
\lambda_{0}=5 \mathrm{mg} / \mathrm{L} \\
\lambda_{2}=15 \mathrm{mg} / \mathrm{L} \\
\lambda_{3}=0.025 \mathrm{mg} / \mathrm{L} \\
x_{50}=1500 \\
\text { cells } / \mu \mathrm{l} \\
k_{5}=0.0 / \text { day } \\
\text { constant } \\
\text { infusion }\end{array}$ \\
\hline
\end{tabular}

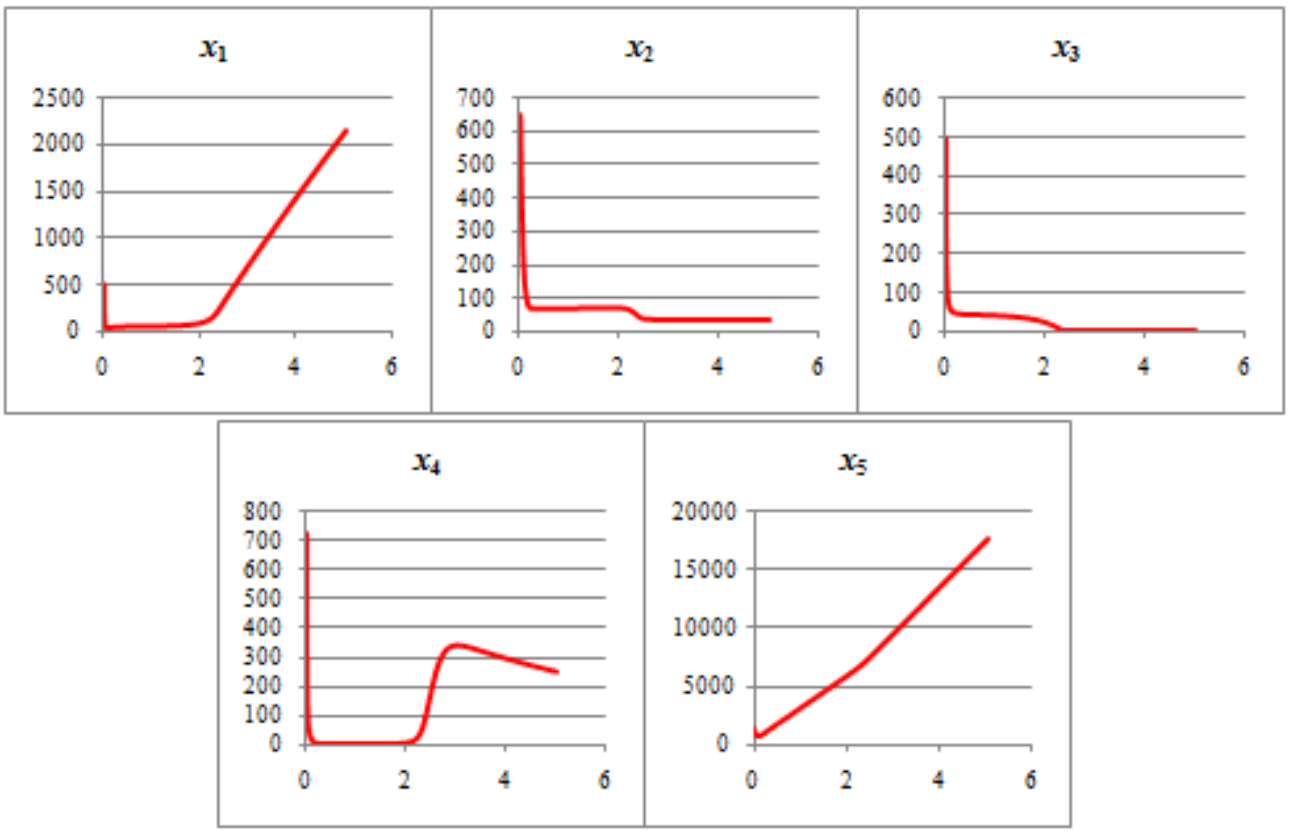

Fig. 1. Simulation results using parametric configuration $P_{1}$ 


\subsection{Simulation Results for Hypothetical AIDS patient \#2}

The hypothetical AIDS patient \#2 is assigned the patho-physiological parametric configuration $\mathrm{P}_{2}$ as presented in Table 2. The simulation results are exhibited in Fig. 2. It can be observed that this patient does have a clinically favorable prognosis under HAART treatment protocol. The AIDS patho - physiodynamics during the given HAART protocol is similar to that for patient \#1, as the patient \#2 also undergoes immune system reconstitution in which the $\mathrm{CD} 4{ }^{+} \mathrm{T}$ cells repopulate. On the other hand, the proliferative activity of the HIV-1 specific CD8 ${ }^{+} \mathrm{T}$ cells appears to be downregulated.

Table 2. Hypothetical AIDS patient parametric configuration $\boldsymbol{P}_{2}$

\begin{tabular}{|c|c|c|c|c|}
\hline $\begin{array}{l}S_{1}=800 / \mathrm{day} / \mu l \\
a_{1}=0.15 \\
/ \text { day } / \mathrm{cell} / \mu l \\
b_{1}=0.005 / \mathrm{cell} / \mu l \\
\alpha_{1}= \\
0.5 / \mathrm{day} / \mathrm{virion} / \mu l \\
k_{1}=0.0005 / \mathrm{day} / \mu l \\
q_{1}= \\
0.00045 / \mathrm{day} / \mu \mathrm{l} / \mathrm{ce} \\
11 \\
\mathrm{e}_{10}=0.0025 \\
\text { cells } / \text { day } / \mu l \\
x_{10}=500 \mathrm{cells} / \mu l\end{array}$ & $\begin{array}{l}S_{2}=800 / \mathrm{day} / \mu l \\
a_{2}=0.03 / \mathrm{day} / \mathrm{cell} / \mu l \\
b_{2}=0.004 / \mathrm{cell} / \mu l \\
\alpha_{2}=0.5 / \mathrm{day} / \mathrm{virion} / \mu l \\
k_{2}=0.005 / \mathrm{day} / \mu l \\
q_{2}= \\
0.00001 / \mathrm{day} / \mu \mathrm{l} / \mathrm{cell} \\
\beta_{1}=1.05 \\
\text { virons } / \mathrm{CD} 4^{+} / \mathrm{day} \\
K_{1}=0.0001 / \mathrm{day} / \mu l \\
e_{20}=0.005 \\
\text { cells } / \mathrm{day} / \mu l \\
\xi_{2}=0.85 \\
x_{20}=400 \mathrm{cells} / \mu l\end{array}$ & $\begin{array}{l}S_{3}=10 / \mathrm{day} / \mu l \\
\beta_{2}=0.0015 \\
\text { virions } / \mathrm{CD} 4^{+} / \text {day } / \mu l \\
\beta_{3}=1.05 \\
\text { virons } / \mathrm{CD} 4^{+} / \text {day } \\
\alpha_{3}= \\
0.027 / \text { day } / \text { virion } / \mu l \\
k_{3}=0.0001 / \text { day } / \mu l \\
e_{30}=0.0001 / \text { day } \\
\eta_{1}=0.25 \\
\xi_{3}=0.001 \\
x_{30}=500 \text { cells } / \mu l\end{array}$ & $\begin{array}{l}S_{4}=10 / \mathrm{day} / \mu l \\
a_{4}=0.35 \\
/ \text { day } / \text { cell } / \mu l \\
b_{4}=0.01 / \mathrm{cell} / \mu l \\
K_{2}=0.0024 \\
/ \text { day } / \mu l \\
k_{4}=0.08 / \text { day } / \mu l \\
e_{40}=0.0002 \\
\text { cells } / \text { day } / \mu l \\
\eta_{2}=0.45 \\
x_{40}=730 \\
\text { cells } / \mu l\end{array}$ & $\begin{array}{l}\mathrm{D}=5000 \text { units } \\
\sigma_{0}=0.5 \mathrm{mg} / \text { day } \\
\sigma_{2}=30 \mathrm{mg} / \text { day } \\
\sigma_{3}=5 \mathrm{mg} / \text { day } \\
\lambda_{0}=5 \mathrm{mg} / \mathrm{L} \\
\lambda_{2}=15 \mathrm{mg} / \mathrm{L} \\
\lambda_{3}=0.025 \mathrm{mg} / \mathrm{L} \\
x_{50}=1500 \\
\text { cells } / \mu \mathrm{l} \\
k_{5}=0.0 / \text { day } \\
\text { constant } \\
\text { infusion }\end{array}$ \\
\hline
\end{tabular}
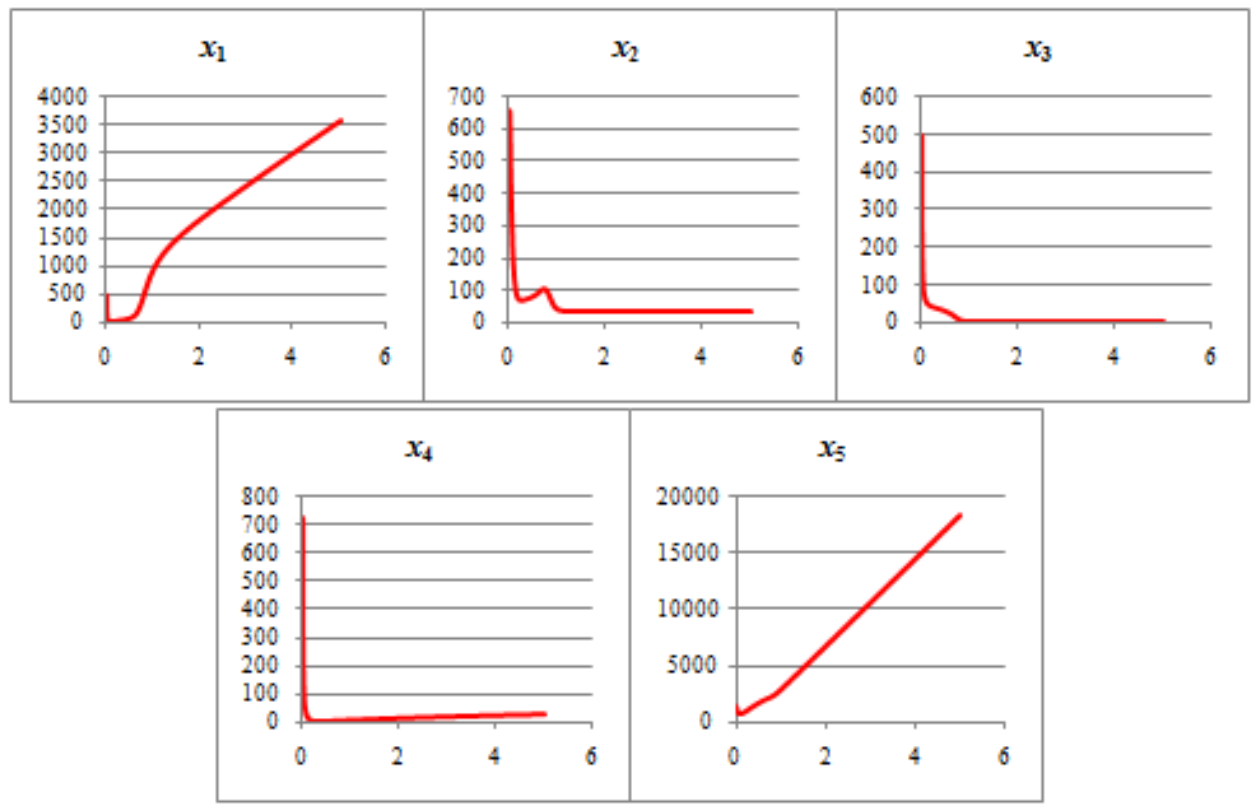

Fig. 2. Simulation results using parametric configuration $P_{2}$ 


\subsection{Simulation Results for hypothetical AIDS patient \#3}

For this scenario, the patho-physiological parametric configuration of hypothetical patient \#3 is as shown in Table 3. It must be noted that HIV-1 AIDS dynamics during HAART treatment protocol in this patient is exacerbated by contributions from latent reservoirs in contrast to the other hypothetical patients. The results of the simulation are shown in Fig. 3. This is a non-equilibrium AIDS configuration simulation as it is evident parametric configuration and by the simulation time profile. The simulation results show that during the given HAART protocol, the hypothetical AIDS patient \#3 experiences annihilation of uninfected $\mathrm{CD} 4^{+} \mathrm{T}$ cells and the HIV-1 specific CD8 ${ }^{+}$ $\mathrm{T}$ cells . Consequently, immune system paralysis occurs as a consequence of the low $\mathrm{CD} 4^{+}$Tcell number density, leading to an exponential increase of the blood plasma HIV-1 viremia. This simulation represents an unfavorable AIDS outcome during HAART.

Table 3. Hypothetical AIDS patient parametric configuration $\boldsymbol{P}_{3}$

\begin{tabular}{|c|c|c|c|c|}
\hline $\begin{array}{l}S_{1}=800 / \mathrm{day} / \mu l \\
a_{1}=0.15 / \text { day } / \text { cell } / \mu l \\
b_{1}=0.003 / \text { cell } / \mu l \\
\alpha_{1}=0.5 / \text { day } / \text { virion } / \mu l \\
k_{1}=0.0005 / \text { day } / \mu l \\
q_{1}= \\
0.00045 / \text { day } / \mu l / \text { cell } \\
\mathrm{e}_{10}=0.0025 \\
\text { cells } / \text { day } / \mu l \\
x_{10}=250 \text { cells } / \mu l\end{array}$ & $\begin{array}{l}S_{2}=800 / \mathrm{day} / \mu l \\
a_{2}=0.03 / \mathrm{day} / \mathrm{cell} / \mu l \\
b_{2}=0.004 / \mathrm{cell} / \mu l \\
\alpha_{2}=0.5 / \text { day } / \text { virion } / \mu l \\
k_{2}=0.005 / \mathrm{day} / \mu l \\
q_{2}= \\
0.00001 / \text { day } / \mu l / \text { cell } \\
\beta_{1}=1.05 \\
\text { virons } / \mathrm{CD} 4^{+} / \text {day } \\
K_{1}=0.0001 / \text { day } / \mu l \\
e_{20}=0.005 \\
\text { cells } / \text { day } / \mu l \\
\xi_{2}=0.85 \\
x_{20}=400 \text { cells } / \mu l\end{array}$ & $\begin{array}{l}S_{3}=10 / \text { day } / \mu l \\
\beta_{2}=0.0015 \\
\text { virions } / \mathrm{CD} 4^{+} / \text {day } / \mu l \\
\beta_{3}=1.05 \\
\text { virons } / \mathrm{CD} 4+\text { day } \\
\alpha_{3}= \\
0.027 / \text { day } / \text { virion } / \mu l \\
k_{3}=0.0001 / \text { day } / \mu l \\
e_{30}=0.0001 / \text { day } \\
\eta_{1}=0.25 \\
\xi_{3}=0.001 \\
x_{30}=500 \text { cells } / \mu l\end{array}$ & $\begin{array}{l}S_{4}=10 / \mathrm{day} / \mu l \\
a_{4}=0.35 \\
/ \text { day } / \mathrm{cell} / \mu l \\
b_{4}=0.002 / \mathrm{cell} / \mu l \\
K_{2}=0.095 / \mathrm{day} / \mu l \\
k_{4}=0.08 / \mathrm{day} / \mu l \\
e_{40}=0.0002 \\
\text { cells } / \text { day } / \mu l \\
\eta_{2}=0.45 \\
x_{40}=730 \mathrm{cells} / \mu l\end{array}$ & $\begin{array}{l}\mathrm{D}=5000 \text { units } \\
\sigma_{0}=1.0 \mathrm{mg} / \text { day } \\
\sigma_{2}=30 \mathrm{mg} / \text { day } \\
\sigma_{3}=5 \mathrm{mg} / \text { day } \\
\lambda_{0}=5 \mathrm{mg} / \mathrm{L} \\
\lambda_{2}=15 \mathrm{mg} / \mathrm{L} \\
\lambda_{3}=0.025 \mathrm{mg} / \mathrm{L} \\
x_{50}=3000 \\
\text { cells } / \mu l \\
k_{5}=0.0 / \text { day } \\
\text { Constant } \\
\text { Infusion }\end{array}$ \\
\hline
\end{tabular}

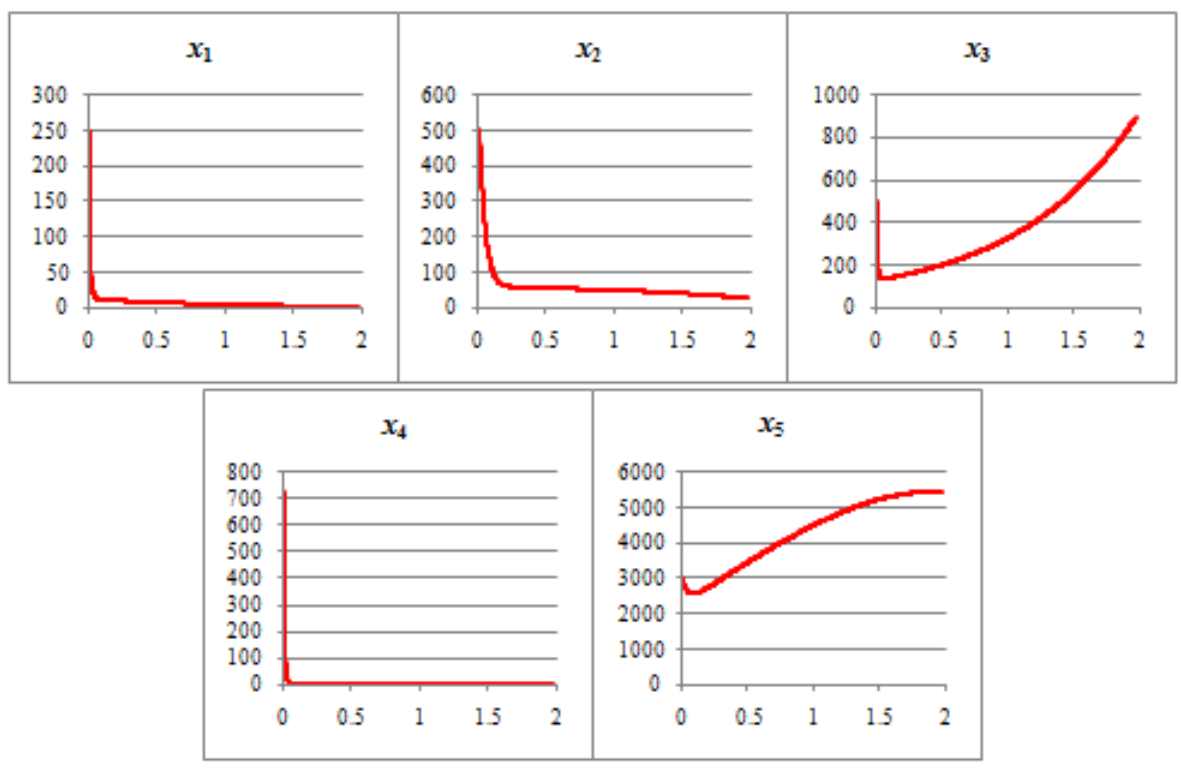

Fig. 3. Simulation results using parametric configuration $P_{3}$ 


\subsection{Simulation Results for Hypothetical AIDS Patient \#4}

In this scenario, the drug input is continuous for 6 months and off for another 6 months until HAART is discontinued. Fig. 4 presents an unsuccessful HAART therapy for a hypothetical AIDS patient with the patho-physiological parametric configuration $P_{4}$ in Table 4 . In this HAART scenario, the plasma HIV-1 virions $\left(x_{3}\right)$ completely overwhelmed the non-infected $\mathrm{CD} 4^{+} \mathrm{T}$ helper cells $\left(x_{1}\right)$ and the HIV-1 specific CD8 ${ }^{+}$cytotoxic T cells $\left(x_{4}\right)$. The HIV-1 infected CD4 ${ }^{+} \mathrm{T}$ cells $\left(x_{2}\right)$ exhibit periodic dynamics and the prognosis for the hypothetical patient is unwholesome (see Nani and Jin $[15,16])$.

Table 4. Hypothetical AIDS Patient Parametric Configuration $\boldsymbol{P}_{\mathbf{4}}$

\begin{tabular}{|c|c|c|c|c|}
\hline $\begin{array}{l}S_{1}=400 / \text { day } / \mu l \\
a_{1}=0.09 / \text { day } / \text { cell } / \mu l \\
b_{1}=0.01 / \text { cell } / \mu l \\
\alpha_{1}=0.5 / \text { day } / \text { virion } / \mu l \\
k_{1}=0.0005 / \text { day } / \mu l \\
q_{1}= \\
0.00045 / \text { day } / \mu l / \text { cell } \\
\mathrm{e}_{10}= \\
0.0025 \text { cells } / \text { day } / \mu l \\
x_{10}=800 \text { cells } / \mu l\end{array}$ & $\begin{array}{l}S_{2}=800 / \mathrm{day} / \mu l \\
a_{2}=0.03 / \mathrm{day} / \mathrm{cell} / \mu l \\
b_{2}=0.004 / \mathrm{cell} / \mu l \\
\alpha_{2}=0.5 / \mathrm{day} / \mathrm{virion} / \mu l \\
k_{2}=0.005 / \mathrm{day} / \mu l \\
q_{2}= \\
0.00001 / \mathrm{day} / \mu l / \mathrm{cell} \\
\beta_{1}=1.5 \\
\text { virons } / \mathrm{CD}^{+} / \mathrm{day} \\
K_{1}=0.0001 / \mathrm{day} / \mu l \\
e_{20}=0.0005 \\
\text { cells } / \mathrm{day} / \mu l \\
\xi_{2}=0.85 \\
x_{20}=400 \mathrm{cells} / \mu l\end{array}$ & $\begin{array}{l}S_{3}=10 / \text { day } / \mu l \\
\beta_{2}= \\
0.0015 \text { virons } / \mathrm{CD}^{+} / \mathrm{day} / \mu l \\
\beta_{3}=1.05 \text { virons } / \mathrm{CD}^{+} / \text {day } \\
\alpha_{3}=0.027 / \text { day } / \text { virion } / \mu l \\
k_{3}=0.0001 / \text { day } \\
e_{30}=0.0001 / \text { day } \\
\eta_{1}=0.25 \\
\xi_{3}=0.001 \\
x_{30}=500 \text { cells } / \mu l\end{array}$ & $\begin{array}{l}S_{4}=10 / \mathrm{day} / \mu l \\
a_{4}=0.35 \\
/ \text { day } / \text { cell } / \mu l \\
b_{4}= \\
0.01 / \text { cell } / \mu l \\
K_{2}=0.0024 \\
/ \text { day } / \mu l \\
k_{4}= \\
0.08 / \text { day } / \mu l \\
e_{40}=0.0002 \\
\text { cells } / \text { day } / \mu l \\
\eta_{2}=0.45 \\
x_{40}=730 \\
\text { cells } / \mu l\end{array}$ & $\begin{array}{l}\mathrm{D}=3000 \mathrm{units} \\
\sigma_{0}=0.5 \mathrm{mg} / \text { day } \\
\sigma_{2}=30 \mathrm{mg} / \text { day } \\
\sigma_{3}=5 \mathrm{mg} / \text { day } \\
\lambda_{0}=5 \mathrm{mg} / \mathrm{L} \\
\lambda_{2}=10 \mathrm{mg} / \mathrm{L} \\
\lambda_{3}=0.025 \mathrm{mg} / \mathrm{L} \\
x_{50}=1500 \\
\text { cells } / \mu \mathrm{l} \\
n=5\end{array}$ \\
\hline
\end{tabular}

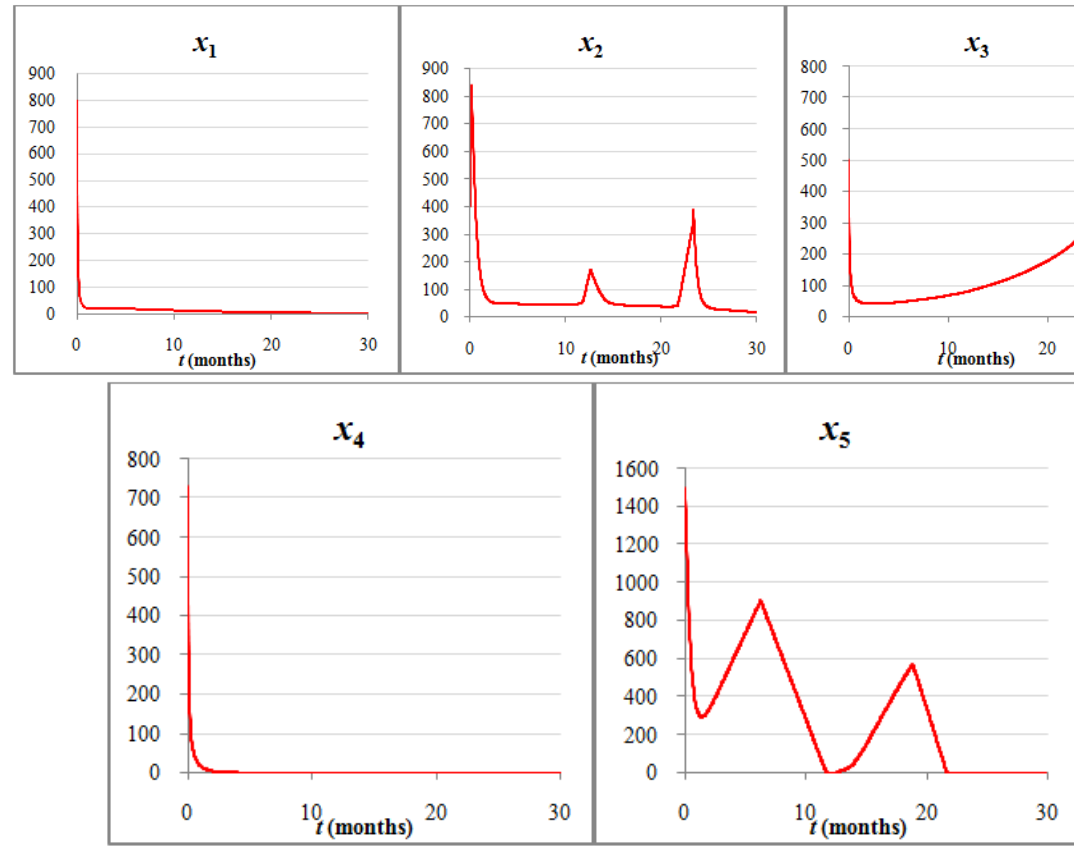

Fig. 4. Simulation results using parametric configuration $\boldsymbol{P}_{4}$ 


\section{Conclusion}

The current paper is elaborate and quantitative attempt to construct medically applicable mathematic models and derive criteria for efficacious HAART protocol for an AIDS patient. The mathematical equations presented in Section 3 are a generalization of the models by Perelson et al. [3], Wodarz and Nowak [5], and Tan et al. [23], and many other authors by incorporating more clinically relevant parameters of HIV-1 patho-physiodynamics. In particular, interleukim-2 activation of both $\mathrm{CD} 4^{+} \mathrm{T}$ cells and $\mathrm{CD} 8^{+} \mathrm{T}$ cells are implicitly incorporated into the model. In this paper, it has been demonstrated that the parameters $\left(a_{1}, b_{1}\right)$ and $\left(a_{4}, b_{4}\right)$ play a significant role in determining the efficacious outcomes of HAART. Clearly, the clinically desirable outcomes are the rest points $E_{4}$ and $E_{3}$. The criteria for the existence of $E_{4}$ and $E_{3}$ are listed respectively in Theorem 5.1, and Theorem 5.4.These theorems give the conditions under which both $E_{4}$ and $E_{3}$ can be temporarily or transiently attained. The criteria for $E_{4}$ and $E_{3}$ to become global attractors are listed in Theorem 6.1 and Theorem 6.2. These theorems, expressed in terms of simple clinically attainable and measurable parameters, give the sufficient conditions for the cure of AIDS. It will be emphasized that there exist other therapeutic criteria but these are intractable and extremely arduous to achieve in a clinical setting.

One essential feature of this research is that the HAART protocol is implemented by using the constant continuous intravenous infusion or constant continuous transdermal infusion of the drug. As such, the criteria derived in this paper are applies to these settings. The mathematical model for intermittent caplet or matrix tablet per oral drug administration can be derived using the nonautonomous version of the model equations involving the drug input function $f(t)$ discussed by Nani and Jin in [7].

The scenarios for clinical failure during HAART due to extreme drug toxicity are represented by the rest points $E_{1}$ and $E_{5}$. The criteria for these therapeutic outcomes are described by Theorem 5.3 and Theorem 5.2, respectively.

In conclusion, it must be emphasized that these theoretical results do not constitute clinical cures unless the results are implemented together with other pharmacological protocols for the AIDS patients. There may exist other theoretical results which are not explicitly discussed in this publication. Nevertheless it is essential to note that mathematical modeling has a place in the quest for the treatment and cure for HIV-1 induced AIDS. Using the techniques of the mathematical modeling and non-linear system analysis, we demonstrated that the activation and kinetic rate constants as well as the rates of influx from latently infected reservoir are important factors in the determination of the prognosis and therapeutic efficacy of HAART.

The future work will involve the construction of mathematical models which will describe an AIDS therapy using HAART in addition to ACI (Active Cellular Immunotherapy) involving interleukim-2. Also, in the future, the mathematical model for HAART will include the roles of mutations such as the delta32 CCR5 on therapeutic outcomes.

\section{Competing Interests}

Authors have declared that no competing interests exist. 


\section{References}

[1] Hess C, et al. HIV-1 specific $\mathrm{CD}^{+} \mathrm{T}$ cells with an effector phenotype and control of viral replication, Lancet. 2004;362:863-866.

[2] Pantaleo G, Fauci AS. New concepts in the immunopathogenesis of HIV infection. Annual Review of Immunology. 1995;13:487-512.

[3] Perelson AS, et al. Decay characteristics of HIV-1 infected compartments during combination therapy, Nature. 1997;387:188-191.

[4] Nowak MA, Bonhoeffer S, Shaw GM, May RM. Anti-viral drug treatment: dynamics of resistance of free virus and infected cell population. J Theor Biol. 1997;184:203-217.

[5] Wodarz D, Nowak MA. Specific therapy regimes could lead to long-term immunological control of HIV. Proc National Acad Sci USA. 1999;96:14464-14469.

[6] Ye P, Kourtis AP, Kirschner DE. Reconstitution of thymic function in HIV-1 patients treated with highly active antiretroviral therapy, Clinical Immunology. 2003;106:95-105.

[7] Nani F, Jin M. Computer simulation of a mathematical model of HAART therapy for HIV1 AIDS, in Proceedings of the International Conference on Bio Medical Engineering and Informatics (BMEI'11). Shanghai, China; 2011.

[8] Lowe SH, Prins JM, Lange JM. Anti-retroviral therapy in previously untreated adults infected with the human immunodeficiency virus type 1: established and potential determinants of virological outcome. Neth J Med. 2004;62:424-440.

[9] Doepel LK. International HIV/AIDS trial finds continuous antiretroviral therapy superior to episodic Therapy, NIH News, National Institute of Health; 2006. Available from: http://www.nih.gov/news/pr/jan2006/niald-18.htm

[10] Bajaria SH, Webb G, Kirschner ED. Predicting differential responses to structured treatment interruptions during HAART. Bulletin of Mathematical Biology. 2004;66:10931118 .

[11] Caetano MAL, Yoneyama T. Short and long period optimization of drug doses in the treatment of AIDS. Anais de Academia Brasileira de Ciências. 2002;74(3):379-392.

[12] Jain S, Tiwary AK, Jain NK. Transdermal delivery of an anti-HIV agent using elastic liposomes: mechanism of action. Current Drug Delivery. 2006;3(2):157-166.

[13] Jin X, et al. An antigenic threshold for maintaining human immunodeficiency virus type 1specific cytotoxic T lymphocytes. Mol Med. 2000;6:803-809.

[14] Lisziewicz J, Lori F. Structured treatment interruptions in HIV/AIDS therapy, Microbes and Infection. 2002;4:207-214. 
[15] Nani F, Jin M. Criteria for annihilation of HIV-1 during HAART therapy. Proceedings of the International Conference on Bioinformatics and Computational Biology 2011 (BIOCOMP'11), Las Vegas, NV; 2011.

[16] Nani F, Jin M. Theoretical Analysis and Simulation of Acute and Chronic Phase HIV-1 Dynamics, British Journal of Mathematics \&Computer Science. 2014;4(11):1450-1479.

[17] Hattaf K, Yousfi N. Dynamics of HIV infection model with therapy and cure rate. International Journal of Tomography and Statistics. 2011;16(W11):74-80.

[18] Pomerantz RJ, Reservoirs of human immunodeficiency virus type 1: the main obstacles to viral eradication. Clinical Infectious Diseases. 2002;34:91-97.

[19] Rong L, Perelson A. Modeling latency infected cell activation: viral and latent reservoir persistence, and viral blips in HIV-infected patients on potent therapy. PLoS Computational Biology. 2009;5(10):e1000533.

[20] Kim H, Perelson A. Viral and latent reservoir persistence in HIV-1 infected patients on therapy. PLoS Computational Biology. 2006;2(10):e135.

[21] Sedaghat AR, Siliciano RF, Wilke CO. Low-level HIV-1 replication and the dynamics of the resting CD4+ $\mathrm{T}$ cells reservoir for HIV-1 in the setting of HAART. BMC Infectious Diseases. 2008;8:2.

[22] Stengel RF. Mutation and control of the human immunodeficiency virus. Mathematical Biosciences. 2008;231:93-102.

[23] Tan WY, Xiang Z. Some state space models of HIV pathogenesis under treatment by antiviral drugs in HIV-infected individuals. Mathematical Biosciences. 1999;156:69-94.

[24] Zaric GS, Bayoumi AM, Brandean ML, Owens DK. Effects of protease inhibitors on the spread of HIV strains, a simulation study. Simulation. 1998;262-275.

[25] Hattaf K, Yousfi N. Two optimal treatments of HIV infection model. World Journal of Modelling and Simulation. 2012;8:27-35.

[26] Hattaf K, Yousfi N. Optimal control of a delayed HIV infection model with immune response using an efficient numerical method. ISRN Biomathematics; 2012.

[27] Koot M, Keet PM, Vos A, Goede R, et al. Prognostic value of HIV-1 syncytium-inducing Phenotype for rate of CD4+ cell depletion and prognosis to AIDS. Ann Intern Med. 1993;118(9):681-688.

[28] Shutt DC, Jenkins LM, Carolan EJ, Stapelton J, Daniels KJ, Kennedy RC, Soll DR. T cell syncytia induced by HIV release. Journal of Cell Science. 111, 1998:99-109. 
[29] Hsu SB. Ordinary differential equations with applications, series on applied mathematics, $2^{\text {nd }}$ edition. World Scientific Press, London, UK. 2013;21:34-35.

[30] Gantmacher FR. The theory of matrices. Chelsea Publishing Co., New York, NY. 1995;300-308.

(C) 2015 Nani and Jin; This is an Open Access article distributed under the terms of the Creative Commons Attribution License (http://creativecommons.org/licenses/by/4.0), which permits unrestricted use, distribution, and reproduction in any medium, provided the original work is properly cited.

Peer-review history:

The peer review history for this paper can be accessed here (Please copy paste the total link in your browser address bar)

www.sciencedomain.org/review-history.php?iid=726\&id=6\&aid=6735 